Voix et Images

voixetimages

\title{
La réception de la littérature canadienne-française en France, au XIX ${ }^{\mathrm{e}}$ siècle
}

\section{Pierre Hébert}

Volume 11, numéro 2 (32), hiver 1986

Michel van Schendel

URI : https://id.erudit.org/iderudit/200558ar

DOI : https://doi.org/10.7202/200558ar

Aller au sommaire du numéro

Éditeur(s)

Université du Québec à Montréal

ISSN

0318-9201 (imprimé)

1705-933X (numérique)

Découvrir la revue

Citer cet article

Hébert, P. (1986). La réception de la littérature canadienne-française en France, au XIX ${ }^{\mathrm{e}}$ siècle. Voix et Images, 11(2), 265-300. https://doi.org/10.7202/200558ar d'utilisation que vous pouvez consulter en ligne.

https://apropos.erudit.org/fr/usagers/politique-dutilisation/ 


\title{
La réception de la littérature canadienne-française en France, au XIX ${ }^{\mathrm{e}}$ siècle*
}

\author{
par Pierre Hébert, University of Toronto
}

Les écrits, venant de France, sur la Nouvelle-France et sur le Canada français, se sont succédé plus ou moins régulièrement à partir de 1545 , date du premier ouvrage français sur le Canada ${ }^{1}$, jusqu'aux nombreuses études actuelles. Le XIX' siècle, pour sa part, s'est avéré singulièrement riche, à la faveur des nombreux voyages qui s'effectuèrent d'un continent à l'autre, de l'intérêt suscité par une littérature française transatlantique naissante, et d'une réanimation de la politique coloniale en France vers 1880.

La littérature canadienne-française du XIX siècle, de son côté, s'est signalée en France dès la parution des Ẽpîtres, satires, chansons, épigrammes et autres pièces de vers de Michel Bibaud, en 1830: il ne fallut que quelques mois pour que ce primeur littéraire eût un écho en France ${ }^{2}$. Témoin le compte rendu partagé d'Isidore Lebrun dans la Revue encyclopédique (juin 1831). Mais si les lettres canadiennes furent commentées de manière discontinue jusque vers 1875 , et plus abondamment après cette date, quel est le système de normes et de valeurs qui a guidé le critique français dans son appréciation? Comment l'a-t-il appliqué à la littérature canadienne-française alors en plein éveil? À ce sujet, on ne dispose que d'informations fragmentaires (comme on le verra dans la première partie de cet article) et, trop souvent, de jugements hâtifs: la France a-t-elle reçu uniformément les œuvres canadiennes-françaises de 1830 à 1900 ? Sinon, quelles ont été les modulations du point de vue français? La France s'est-elle constamment proposée comme modèle? Enfin et surtout, quel a été le système axiologique qui a soutenu l'exercice de son jugement littéraire?

Cette étude souhaite répondre à de pareilles questions. Dans le contexte des relations littéraires entre la France et le Canada français, il s'agira de reconstituer l'horizon d'attente français, de montrer comment les œuvres et l'idée même d'une littérature venant de la «France transatlantique» ont été reçues, et d'expliquer, le cas échéant, les mutations d'horizon et de réception. À cette fin, notre objet d'étude, la méthode de travail choisie, la période élue de même que le corpus dépouillé appellent certaines précisions.

Notre objet, la littérature canadienne ou canadienne-française selon les appellations de l'époque, comprendra la poésie, le roman et le théâtre, encore que nous nous permettrons quelques regards sur d'autres genres: comment

* Cette recherche a été faite grâce à une subvention du Conseil de recherches en sciences humaines du Canada. 
ne pas parler de l'Histoire de Garneau? D'autre part, nous aurons l'occasion, lors de la section qui portera sur l'horizon d'attente proprement dit, de soulever dans le détail plusieurs questions relatives à notre méthode de travail. Mais il est sans doute apparu, d'entrée de jeu, que cette recherche se loge à l'enseigne de la théorie de la réception, de H.-R. Jauss. Remettant donc à plus tard certains scrupules théoriques, nous poserons simplement pour l'instant que, par horizon d'attente, nous entendons avec Jauss le système de références objectivement formulable ${ }^{3}$ qui balise la réception des œuvres, et qui nous guidera dès lors dans la compréhension de l'accueil fait à la littérature canadienne-française. Quant à la période choisie, elle s'ouvre en 1830 pour des raisons évidentes. Mais pourquoi terminer en 1900 ? Cette coupe tient essentiellement au fait que le discours critique français se transforme à cette époque. La fin du XIX $\mathrm{X}^{\mathrm{c}}$ siècle et le début du XX $\mathrm{X}^{\mathrm{e}}$ sont marqués, on le sait, par la parution des premières études sur la littérature du Canada français, en l'occurrence celles de Virgile Rossel et de Charles ab der Halden 4 . Le tournant du siècle signale le début d'une appropriation des lettres canadiennes par l'institution critique française.

Enfin, et plus longuement, des indications sur notre corpus de textes de critique. Celui-ci a été établi à partir d'un inventaire des journaux, revues et chapitres (ou sections de chapitres) de livres parus en France, et portant sur notre objet d'étude. Sans prétentions d'originalité bibliographique - outre quelques trouvailles fortuites - nous avons dépouillé systématiquement les travaux de David M. Hayne, Armand Yon et Gérard Tougas 5 ; nous avons également puisé quelques indications chez Gustave Lanctôt ${ }^{6}$. C'est donc à l'aide de cet ensemble de quelque soixante-quinze articles (journaux et revues) et vingt volumes (chapitres ou sections) que nous pourrons décrire l'accueil français?. En outre, nous obtenons un corpus de textes assez homogène: voyageurs, gens cultivés, dilettantes de la littérature caractérisent, à peu d'exceptions près, les auteurs qui à cette époque ont fait de la littérature canadienne-française l'objet de leurs écrits. C'est dans un sens qui a peu à voir avec la «critique professionnelle " que nous parlerons du lecteur français, du critique et, partant, de réception critique.

Dans une première partie ${ }^{8}$, la plus importante, nous circonscrirons l'attente du lecteur français et caractériserons la réception de la littérature canadienne-française; comme cette réception n'est pas uniforme de 1830 à 1900, nous l'étudierons en trois volets: avant 1880 , puis les années 80 et la réception variable de Fréchette et Crémazie et, enfin, après 1880 . Dans une courte deuxième partie, nous proposerons, en guise de conclusion, quelques hypothèses quant au changement de réception que nous aurons pu observer. 


\section{La réception de la littérature canadienne-française au $\mathrm{XIX}^{\mathrm{e}}$ siècle.}

Il est bien vrai que nous autres, lecteurs ou critiques, nous lisons avec un passé. De même que tout homme a un passé, tout lecteur a un passé d'habitudes, de culture, de références, qui lui donnent souvent quelques clés pour déchiffrer les ceuvres mais qui lui en otent parfois plus qu'ils ne lui en rapportent lorsqu'il se trouve devant une ouvre originale, irréductible à celles qui l'ont précédée ${ }^{9}$.

\section{Pierre de Boisdeffre}

Voilà ce qu'écrivait un critique français, dans la meilleure tradition herméneutique, mais bien avant que l'École de Constance ne connaisse l'audience qui dure encore aujourd'hui. Voilà également qui montre bien combien l'esthétique de la réception repose sur une sorte de sens commun, ce qui n'est pas un moindre mérite. Toute interprétation d'un texte doit donc commencer par une réflexion de l'interprète sur les idées préconçues qui résultent de la "situation herméneutique" où il se trouve ${ }^{10}$. Le coup d'envoi est ainsi donné: reconstituer, à un second degré, cette situation herméneutique du lecteur français vis-à-vis de la littérature canadienne. N'allons pas croire, pourtant, qu'aucun problème ne grève l'esthétique de la réception. Elle présuppose, par exemple, jusqu'à un certain point, un type de lecteur qui participe de «l'humaniste libéral» toujours prêt à voir ses croyances modifiées ${ }^{11}$; elle implique un souci constant, d'autre part, de vouloir rendre l'cuvre cohérente; elle ne tient pas compte du degré de détermination opérée par le texte lui-même, en sus des déterminations normatives du lecteur; enfin, elle n'inclut pas, dans le processus de communication littéraire, la production du texte ${ }^{12}$. En deça des limites que nous nous sommes données, cette approche trouvera cependant sa fécondité dans la mesure où, tout d'abord, elle permet de distinguer l'horizon d'attente de la réception critique, et de caractériser l'un pour comprendre l'autre.

Le "modèle" d'analyse de Jauss reste cependant idéal, et il doit être moulé sur les circonstances et l'objet de travail. C'est ainsi qu'en posant un certain nombre de "questions" aux textes de notre corpus, nous sommes arrivé à cerner les constituantes des horizons d'attente et de réceptions qui nous intéressent ici $^{13}$.

Notons en passant le pluriel des mots horizons et réceptions; en effet, on ne peut parler d'un seul horizon d'attente pour la période qui va de 1830 à 1900 , ni d'une seule réception. L'étude des textes nous a plutôt conduit à en dégager deux, posant les années 1880 comme moment de transformation du système des références des lecteurs. Entre l'étude de ces deux réceptions, nous nous arrêterons assez longuement sur l'accueil fait aux œuvres de Louis Fréchette et d'Octave Crémazie, particulièrement vers 1877-1881, afin de bien faire apparaître les mutations qui marquent cette période. 


\subsection{La première réception des lettres canadiennes-françaises, 1830-1880.}

\subsubsection{L'horizon d'attente}

Dans une série de trois articles importants, parus dans l'Union et intitulés «De la littérature au Canada. Charles Guérin, roman de mœurs, par M. Pierre Chauveau (Québec)", Adolphe-Louis de Puibusque établit, comme en une sorte de concentration de toute la tendance critique de 1830 à 1880 , les principales normes qui guideront la lecture des ouvres canadiennesfrançaises. Nous pouvons le poser comme représentatif de toute cette époque. De Puibusque, lui-même écrivain, possède un jugement nuancé; il est l'auteur d'une Histoire comparée des littératures espagnole et française et il a séjourné au Canada.

Comme s'il avait pressenti l'importance de la réception des œuvres, de Puibusque s'interroge tout $d$ 'abord sur la manière dont on doit accueillir les ouvrages extérieurs à la littérature-mère: L'imitation a une si large part aux ceuvres de l'esprit humain, écrit-il, que tout ce qui semble échapper à l'influence traditionnelle excite un vif étonnement ${ }^{14}$. Mais, se hâte-t-il d'ajouter, lorsque cette production éclôt "loin de l'astre central", l'admiration, si elle doit venir, aura d'abord à triompher du doute [...] (id.). Comme pour renforcer cette difficulté de recevoir favorablement les productions canadiennes, de Puibusque, dans la livraison du 30 juillet, cite un long commentaire de L.-A. Olivier qui s'interroge sur la possibilité des lettres canadiennes d'intéresser l'Europe, courtisane blasée qui ne saurait goûter les émotions douces et tranquilles, mais qu'il faut exciter par des émotions fortes, des portraits nouveaux ${ }^{15}$. Mais ne nous y trompons pas, l'argumentation de de Puibusque est dialectique: il n'a posé cette difficulté que pour la résoudre avec fermeté, dans les termes suivants:

Cente Europe [...] est accessible aux émotions douces, aux pensées chastes, aux nobles enthousiasmes, à tout ce qui est généreux, noble et vrai $[\ldots]$.

Ah! de grâce, ne les croyez pas, vous qui êtes si purs encore! montrez-vous à ses yeux tels que vous êtes, ne prenez conseil que du beau ciel qui vous éclaire, n'écoutez que ces voix de la terre natale qui s'élèvent si sublimes et si naïves autour de vous!

[...] Parler de vous, c'est parler de nous, puisque nous sommes deux fois frères, et comme hommes et comme français; ne supposez donc pas que notre intérêt puisse jamais vous manquer». (L'union, 30 juillet 1855, p. 3)

De Puibusque jette en vérité une programmation de lecture qui sera dominante jusque vers 1880 . Examinons-en, à ce stade-ci, les principaux traits.

\section{Les modèles français}

Contrairement à toute attente, le modèle français n'est jamais posé comme un idéal à atteindre. Les lecteurs qui l'évoquent face à l'œuvre cana- 
dienne le rejettent aussitôt, favorisant la simplicité et la çouleur locales. Albert Lefaivre, qui s'est adonné à la critique littéraire et quii a emprunté, à l'occasion, les pseudonymes de J. Guérard et de Grünewald, fut consul de France au Canada, de 1875 à 1881 . Dans sa Conférence sur la littérature canadienne, il écrit, contre la tendance romantique de la poésie de l'époque:

Certains poètes prennent Victor Hugo pour modèle [...]. Cette magnificence est un peu dépaysée dans le pays des Hurons; plus de simplicité siérait mieux dans une société jeune encore, étrangère aux plaisirs du dilettantisme, c'est-à-dire des esprits blasés. [...] Fils de fermier, pour la plupart accoutumés à la vie rustique, ils ne sont jamais sérieusement atteints de notre spleen et n'en ont pas la distinction ${ }^{16}$.

Les Canadiens français appartiennent à un tout autre univers; et ce n'est pas avant 1878 qu'on entreverra la possibilité de suggérer le modèle français.

\section{Prédominance du contenu sur la forme}

La forme des œuvres canadiennes-françaises, à cette époque, n'offre rien de particulièrement avant-gardiste, c'est le moins qu'on puisse dire. Mais le lecteur français évite bien de monter ce fait en épingle, et préfère exhausser les vertus du "fond». E. F. ${ }^{17}$ pose bien le dilemme:

Les Canadiens, trop sensibles peut-être aux appréciations quelquefois sévères de certains touristes plus affectés de la forme parfois surannée de quelques uns [sic] de leurs écrits que par la solidité du fond et le souffle patriotique qui les inspire, ont bien de la peine à se décider à braver la critique parisienne. [...] Si les lettres du Canada ne jettent pas le même éclat que chez nous, elles n'en appartiennent pas moins à la France, au double point de vue de la langue, des sentiments qu'elles expriment et de l'histoire ${ }^{18}$.

\section{Le vrai, le naturel, le simple}

La prédominance du contenu, associé à la simplicité, conduit tout naturellement le lecteur à goûter la vérité des situations, l'exactitude des descriptions, conforme en cela à l'objectif même que s'étaient fixé les écrivains canadiens de l'époque, les romanciers surtout. La littérature apparaitra dès lors comme le portrait fidèle du Canadien français aux yeux d'une France qui, il faut bien le dire, ne saura guère de choses de lui avant les années 1880-1890.

\section{L'utilisation de la langue française}

Nous étions loin de croire qu'un Canadien de naissance [...] pût être capable d'écrire, en très-bon français, des poésies $[\ldots]^{19}$; cet étonnement de J. Varech à propos de Louis Fréchette et de son recueil Pêle-mêle n'est pas le seul du genre, bien au contraire. Écoutons, par exemple, Camille Doucet, secrétaire perpétuel de l'Académie française, lors de son discours à l'occasion de la remise du prix Montyon: C'est en français, Messieurs, qu'on écrit. qu'on parle et qu'on pense dans ce pays jadis français, que nous aimons et qui 
nous aime ${ }^{20}$. Aussi se gardera-t-on de se montrer sévère pour cette langue, le seul enthousiasme de la voir vivante suffisant largement; si le style lui-même laisse à désirer, on ne s'inquiétera pas outre mesure.

\section{La francité}

Si on s'attend à ce que le Canadien français se peigne tel qu'il est, dans sa simplicité et son naturel, ce n'est pas pour le considérer comme un être autonome, voire isolé, sans filiation française. Bien au contraire! Le lecteur français se montre extrêmement perméable à toute manifestation de francophonie, ou mieux, de francophilie. Le principe des vases communicants fonctionne ici à plein. L'on espère une sympathie, un amour pour la France. Ayant rencontré P.-J.-O. Chauveau, Jean-Jacques Ampère, le fils du grand savant, écrit ce que ses compatriotes espèrent bien pouvoir lire: J'ai appris de lui, ce qui m'a été confirmé par d'autres, combien la population canadienne est occupée de la France ${ }^{21}$. Anticipons sur la réception des œuvres ellesmêmes, pour citer ce jugement sur l'Histoire du Canada, de Garneau, vue par ce même Ampère: $M$. Garneau, qui a bien voulu être mon obligeant cicérone, a écrit une histoire du Canada, la seule complète, fruit de recherches consciencieuses et animée d'une sympathie sincère pour la France. L'histoire du Canada est un complément de notre histoire (Id., p. 115).

\section{Patriotisme, catholicisme et autres vertus}

La voie commence à se déblayer pour faire ressortir les critères du lecteur français: une littérature canadienne restera française et patriote (Lefaivre, Conférence sur la littérature canadienne, p. 19) d'une part, et son appartenance outre-atlantique, d'autre part, rappellera à la fois le génie du christianisme et le génie de la France ${ }^{22}$. $\mathrm{A}$ ces deux critères prédominants s'ajoutera parfois l'aspect moral du texte: est-il nécessaire de rappeler que les prix Montyon sont une sorte de "prix de vertu» décerné aux ouvrages utiles aux mœurs?

\section{L'inspiration, l'élévation de la pensée, le souffle...}

Toutes ces qualités virtuelles du fond de l'œuvre émanent de cette muse vaporeuse, indéfinissable, que le critique appellera souffle, verve, inspiration (principes tributaires le plus souvent des grands espaces canadiens).

Nous venons de prendre contact avec la grille de lecture du «critique» français. Nous pouvons ainsi passer en revue les principales œuvres canadiennes jusqu'à l'invasion - le mot n'est pas trop fort - de la presse française par Louis Fréchette, ce qui explique la place privilégiée, avec Crémazie, que nous lui accorderons. Il ne serait pas inutile, cependant, d'essayer de déceler dans quelle optique les lettres canadiennes-françaises ont été perçues à l'origine, et de quelle manière le lecteur nommait alors cette littérature naissante. Le contexte de saisie des œuvres devrait y gagner en clarté. 


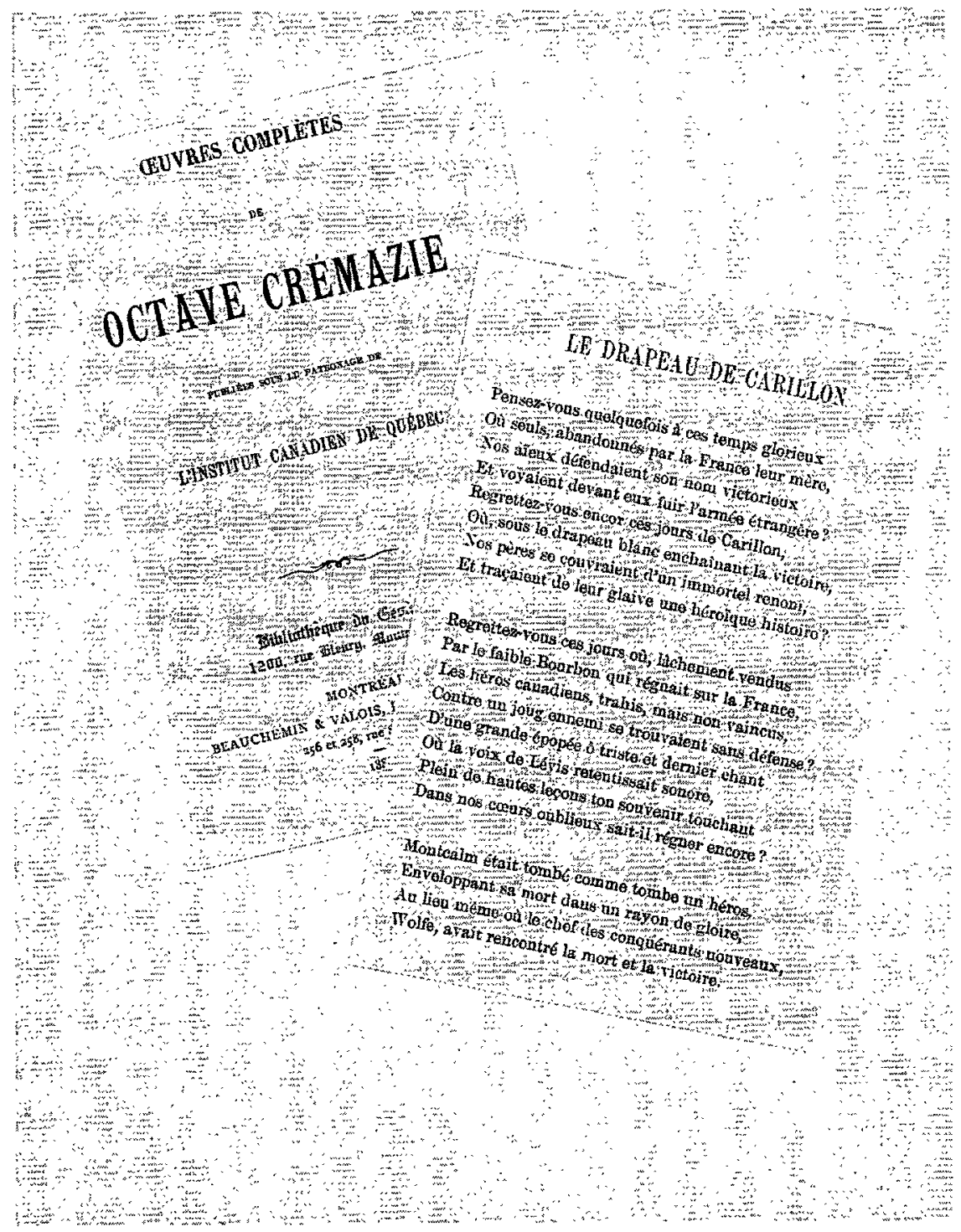




\subsubsection{La réception des ceuvres}

\section{Une curiosité initiale}

Disons-le: à cette époque, on connaît peu et fort mal, le Canada. Le nombre d'erreurs que nous avons pu relever indique combien l'on est loin de dominer son sujet. On pourra lire «Battre» «Mermette», «Fléchette»; "Tableau de la rivière Duelle», "Drapeau de Carillon», «Promenade de trois morts»; Eugène Réveillaud, dont on reparlera, citera De Cazes parmi les écrivains canadiens, Jacques Feyrol parlera de l'épisode de la Capricieuse en 1866 , et nous en passons... L'ouvrage de Lebrun, en $1833^{23}$, arriva dans ce climat d'ignorance et contribua pour bien peu à changer les choses. Des Canadiens français qui s'obstinent à ne pas mourir? Alfred de Vigny déclara qu'à Paris on n'était pas au courant (Ménard, Xavier Marmier..., p. 21).

Curiosités, donc, que ces œuvres qui arrivent d'une race dont Louis Hémon dira qu'elle ne sait pas mourir. Les Épîtres... de Bibaud apparaîtront donc curieux pour les bibliophiles ${ }^{24}$, 1 'Histoire du Canada de Garneau, un livre curieux à tous égards ${ }^{25}$. L'exclamation de Marie Fontenay (Quelle perte pour la France que celle de ce pays, le plus pittoresque et le plus accidenté de la terre! $\left.{ }^{26}\right)$, n'aurait pu être poussée par le directeur de cette même vénérable Gazette de France: Henri de Lourdoueix, manifestant une surprise dont le revers est l'ignorance, avoue:

Notre ignorance sur tous les lieux du Nouveau-Monde qui se trouvent en dehors de l'expansion américaine et de la route des chercheurs d'or est telle, qu'en rappelant aujourd'hui l'attention publique sur le Canada [par l'entremise du volume de Barthe], les faits que nous allons présenter seront pour la généralité de nos lecteurs, comme ils l'ont été pour nous-même, une révélation presque aussi surprenante que le fut pour l'Espagne la découverte du Mexique par Fernand Cortès ${ }^{27}$.

Edmond Farrenc, une vingtaine d'années plus tard, s'étonnera encore de l'ignorance, quand ce n'est pas l'indifférence de ses compatriotes à l'égard du Canada français. C'est jüstement à cette époque que la curiosité se muera en intérêt réel et durable.

\section{Le choix d'un nom}

La littérature existe-t-elle vraiment au Canada français? Bien téméraire qui aurait voulu répondre à une question qui, encore au début de notre siècle, divisait les esprits. Plus modestement, demandons-nous comment cette littérature était nommée par le lecteur français.

Par la force des choses, vers 1830 , on ne pouvait parler de littérature canadienne, ou canadienne-française, même si le chapitre XII du Tableau statistique... de Lebrun s'intitule: «Journaux, Littérature". A partir de 1855 , jusqu'en 1880 , on ne réussira pas à s'arrêter sur une dénomination. Se présenteront les appellations suivantes: "littérature française au Canada" (de 
Puibusque, l'Union, 1855; Paul de Cazes, dans Le Monde ${ }^{28}$, écrit d'abord "la Littérature française au Canada» comme titre d'un article, celui du 26 février, puis, à partir du 17 avril, se tourne vers "la littérature au Canada»); "lettres au Canada» (Edmond Farrenc,Le Constitutionnel, 5 mars 1874); « une littérature française» (E. Farrenc, au même endroit; Raoul Frary, Journal officiel de la République française ${ }^{29}$, reproduit dans la Revue de Montréal, janvier 1879, p. 6); «Littérature canadienne» (Marie Fontenay, Gazette de France, 1855; H. de Lourdoueix, Gazette de France, 1855; P. de Cazes, Le Monde, 6-7 août 1877; Lefaivre, Conférence...., 1877, passim; Frary, Journal officiel de la République française, passim). L'appellation "littérature canadienne» tend cependant à s'imposer, ce qui n'étonne pas. Anticipons pour dire ici que l'étiquette «littérature canadienne" durera jusqu'à la fin du siècle, et même plus loin.

\section{"Histoire du Canada", de Garneau}

Comment furent reçues les œuvres créées au Canada? C'est ce que nous allons maintenant examiner, en donnant préséance à celles qui ont été les plus commentées. L'Histoire du Canada et Charles Guérin méritent ainsi une place à part. Notons en passant une difficulté méthodologique: contrairement à la critique canadienne-française, qui s'efforce de rendre compte de quelque manière de la majorité des oeuvres parues au Canada, la critique française, elle, peut se permettre d'être sélective, surtout à cette époque. De plus, ce ne sont pas toutes les œuvres qui sont connues du lecteur français. Vides, absences, esquives, peuvent être par conséquent pleins de sens.

L'on se rappellera en quels termes $\mathbf{J} .-\mathrm{J}$. Ampère a parlé de l'œuvre historique de Garneau, et que Lefaivre qualifiera, en 1874, du plus beau travail dans ce genre ${ }^{30}$. On a reconnu d'emblée la valeur intrinsèque de l'ouvrage, premier en son genre, bien documenté et même utilisé par des historiens français. Plus de vingt ans après son voyage au Canada, Théodore Pavie célébrera encore les vertus de cette œuvre, écrite dans notre langue et empreinte de ce sentiment de sympathie filiale pour la France (Revue des Deux Mondes, juillet 1853, p. 280). Le lecteur n'a de cesse d'observer que ce monument représente une pièce d'identité de la survivance française et de l'amour pour la France.

Il faut donner une place à part à Edmond Farrenc, et au premier de ses articles du Constitutionnel, celui du 5 mars 1874. Farrenc parlè d'une école historique au Canada français, qui compte parmi ses plus brillants adeptes M. Garneau, l'abbé Ferland et l'abbé Casgrain (repris dans le Journal de I'instruction publique, avril 1874, p. 57). Ces trois historiens, poursuit-il, malgré des divergences de manière, se ressemblent tous en ce qui concerne le fond: leurs pages offrent des traits d'audace, d'abnégation, d'héroisme et de foi chrétienne (id.). Ces ouvrages, dédiés au souvenir de la France et de ceux de nos compatriotes qui ont illustré notre race sont un patrimoine commun dont nous avons le droit de réclamer notre part (id.). Chacun de ces historiens exhibe des vertus particulières, et Garneau, lui, est fort remarqué par la vivacité du récit et l'élévation de la pensée (id.). 
Est-ce à dire que l'Histoire du Canada n'a eu droit qu'à des accolades? Oui, à une faible exception près: Moreau, dans Le Correspondant, après avoir accordé l'importance usuelle à la survivance de la nationalité française au Canada, trouve Garneau injuste et sévère à l'égard du catholicisme; il prend d'ailleurs lui-même la relève pour en exalter les bienfaits.

\section{«Charles Guérin", de P.-J.-O. Chauveau}

Pour un lecteur français en appétit du «Canada tel quel», le roman Charles Guérin, de loin le plus commenté en France, offre un plat de choix: simplicité, naturel, «vérité», tout y est:

$C$ 'est dans cette ligne que $M$. Pierre Chauveau s'est sagacement placé: appliquant toute son étude aux hommes et aux choses qui sont sous ses yeux, il a peint le Canada tel qu'il est et avec un pinceau Canadien; à ne considérer son travail que comme un nouvel acte d'initiative, il devrait être mentionné avec distinction et recevoir le même accueil que les hardis et spirituels essais de MM. de Gaspé, Angers, de Boucherville, Petitclair, Eugène L'Écuyer, Doutre, Patrice Lacombe; mais il a d'autres mérites; il est vrai et moral; il promet donc d'être doublement utile. Ce n'est pas, comme dans René, le vague des passions, tourment d'une jeunesse oisive; c'est la vie réelle avec ses incertitudes, ses calculs, ses espérances, ses mécomptes. (de Puibusque, L'Union, 30 juillet 1855)

Le jeu est étalé non seulement sur les expectatives, mais également sur les modèles rejetés pour apprécier le roman. Mais Charles Guérin n'offre-t-il aucune faiblesse? Bien sûr que non: la critique aurait à signaler plus d'une imperfection dans son roman, si elle l'examinait du point de vue des conventions européennes (id.). Ainsi, le plan final de fondation agricole et l'intérêt inégal du roman. Mais justement, les conventions européennes sont repoussées, ce qui autorise une vision sympathisante de Charles Guérin:

[...] gardons-nous donc de nous montrer trop exigeans [sic] envers un jeune auteur qui nous donne du premier coup un paysage neuf, des scènes de moeurs instructives et une action attachante. Charles Guérin est mieux qu'un bon livre, c'est un bon exemple, il excitera l'émulation de nos anciens compatriotes et nous ne tarderons pas sans doute à voir d'autres anneaux ajoutés à la chaîne qui doit réunir à la fois les esprits et les coeurs des deux France.(id.).

L'étalon européen mis à l'écart, la fonction sociale du texte s'affirme d'autant mieux que le fond canadien efface une certaine pauvreté de l'œuvre. À la limite, les jugements iront jusqu'à renverser l'ordre des choses, généreux à loisir pour louer non seulement le fond, mais le style même! Manoël de Grandfort, qui avait signé de son nom de fille, Marie Fontenay, l'article déjà mentionné de la Gazette de France du 27 février 1855, de même que la 
première édition de L'Autre monde, reprend sa «lettre à Monsieur de La Rochefoucauld" en y ajoutant cette note:

Charles Guérin, par monsieur $P$. $O$. Chauveau, est une oeuvre toute canadienne qui se recommande par les qualités de style les plus brillantes et les situations les plus vraies et les mieux senties. Il y a dans ce livre, où éclate à chaque page la plus véhémente sympathie pour la France et tous ses souvenirs, des descriptions vraiment enchanteresses, quoique pourtant fidèles, des bords du fleuve Saint-Laurent. (L'Autre monde, p. 270-271, n.2)

A la faveur de cette attitude sympathisante, Charles Guérin sera à l'abri de toute critique négative. Encore en 1878, Raoul Frary défendra le roman de Chauveau avec les mêmes arguments que de Puibusque. Tout n'est pas parfait dans cette œuvre, au contraire. Mais sa lecture, de l'aveu de Frary, exige des dispositions particulières:

Il ne faut pas l'aborder avec cette attention critique et cette délicatesse nerveuse qui nous rend sensible aux moindres taches. Il y a ici des taches et des défauts; [...]. Mais dépouillons-nous, pour un instant, de ce goût raffiné et irritable que nous a fait la lecture de nos chefs-d'oeuvre européens. Laissons-nous aller sans résistance au courant de cette narration limpide; laissons-nous toucher par ces scènes d'un pathétique si naturel; laissons-nous amuser par ces peintures naives de la vie de province et de la vie rustique; il nous suffira, pour être émus et charmés, de ne pas nous mettre en défense. (Journal officiel de la République française, reproduit dans la Revue de Montréal, janvier 1879, p. 8-9)

Certes, le rejet des "chefs-d'œuvre» n'étonne pas chez Raoul Frary qui signera, en 1885 , un ouvrage fustigeant l'enseignement classique au profit d'une approche plus vivante. C'est le moment de noter également que cette attitude de disponibilité vis-à-vis des lettres franco-canadiennes exige que le lecteur soit "charmé» plutôt que d'exercer son jugement critique; sans anticiper, disons simplement qu'on aura l'occasion de voir la fortune de ce mot, "charme» en l'occurrence, lors de l'épisode Louis Fréchette. Mais revenons à Charles Guérin: quand on a lu ce roman, poursuit Frary, on croit avoir vécu au Canada, tant les lieux et les gens sont décrits avec netteté, tant il y a de vie et de vérité dans ce récit sans prétention (id., p. 9).

Le seul juge sévère que ce roman ait connu - faut-il s'étonner que ce soit un consul doublé d'un critique littéraire? - fut Lefaivre qui, à l'égard de Chauveau, de Marmette, écrit: c'est partout, et sous mille formes diversement monotones, le manichéisme (Conférence..., 1877, p. 32). N'épargnant rien ni personne, Lefaivre poursuit, incluant les auteurs dramatiques: tout en eux est banal et superficiel [...] (id., p. 33). Pareils jugements annoncent la réception des années 80 . 


\title{
"Le Canada reconquis par la France», de Barthe
}

Publié en France, le Canada reconquis par la France n'a pas tardé à susciter de l'intérêt. Le baron de Lourdoueix, après avoir avoué, comme nous l'avons déjà constaté, son ignorance au sujet du Canada, parle en ces termes du livre "remarquable" et de la "mission" de Barthe: [...] il est impossible de lire ce livre et de ne pas se sentir pris par le tableau qu'il nous trace de cette contrée, des vertus et l'esprit de ses habitans. [ sic]. D'un même souffle, il poursuit:

\begin{abstract}
Hâtons-nous de dire que le but de ce livre n'est pas de provoquer une conquête militaire du Canada par la France: la tâche qu'a entreprise $M$. Barthe, c'est de renouer les liens moraux qui attachaient la France à cette ancienne partie d'elle-même; c'est de créer des rapports sympathiques qui consoleraient nos frères d'Amérique, et de les faire participer, par des communications régulières, à ce grand foyer intellectuel d'où est partie la littérature canadienne qui brille d'un vif éclat dans le Nouveau Monde. (Gazette de France, 1855)
\end{abstract}

L'ouvrage arrive donc à point, et les "rapports sympathiques" ont ici tout leur poids. Voilà pourquoi, sans doute, Pierre-Sébastien Laurentie, directeur de L'Union, n'a pas goûté la première partie de l'ouvrage de Barthe, où l'histoire cède à des procédés un peu trop passionnés; la deuxième partie, en revanche, simple exposé de la situation du Canada, touche davantage ce lecteur à la recherche d'un tableau des mours, des habitudes et des lois de notre vieille colonie, et, dès lors, a plus de chance de remuer les sympathies de la France (L'Union, 26 août 1855). Dans le premier de ses deux articles consacrés au Canada reconquis, Laurentie n'avait pas manqué de manifester l'émotion patriotique que lui avait inspirée la lecture de ce livre.

La réception de ces trois ouvrages (les plus commentés, d'ailleurs, jusque vers 1880 ) conjugue donc la perception de la littérature canadienne tout entière: louée pour ses vertus françaises, tant sur le plan de la langue que par sa participation à l'âme française, l'œuvre sera reçue favorablement, nonobstant certaines faiblesses de forme; ce qui compte d'abord, c'est la communauté de sentiment. Pour fermer cette première tranche chronologique, passons maintenant à la réception d'œuvres mineures en ce qui a trait à leur importance accordée en France.

\section{Les cuvres moins commentées}

Disons tout de suite que la majorité ont bénéficié de l'accueil sympathique dont nous avons fait état. L'adjectif attachants utilisé par Edmond Farrenc pour qualifier les Mémoires et les Anciens Canadiens de PhilippeAubert de Gaspé doit donc être pris littéralement (Le Constitutionnel, 5 mars 1874 , repris dans le Journal de l'instruction publique, avril 1874, p. 58). Et Farrenc de qualifier le Seigneur de Saint-Jean-Port-Joli d' Homère inconnu au style qui n'est pas sans charme [...]. D'autre part, dans Le Monde, à 
partir de la livraison du 6-7 août 1877, paraîtra en feuilleton Jean Rivard; une note liminaire indique fort bien ce qu'on y trouvera:

Nous sommes persuadés que nos lecteurs goûteront ce récit, d'un caractère simple, plein de sentiments élevés, où sont retracés d'une façon intéressante et dans un style naturel et vivant les efforts et les succès d'une lutte chrétienne contre la pauvreté.

Le cadre où le récit est placé lui donne un intérêt particulier pour les lecteurs français. Le Canada, séparé de la France, est resté une terre française et catholique. Sa prospérité merveilleuse, qui a pour raison la vertu de notre foi et les meilleures qualités de notre race, est une joie et un honneur pour la mère patrie.

Suite à cette note anonyme, Paul de Cazes ajoute cet autre aspect qui nous est désormais familier: Ce qui donne à mes yeux une grande valeur au livre de M. Gérin-Lajoie, c'est que tout y est de la plus parfaite exactitude.

Mêmes éloges du côté de la poésie, particulièrement, on le verra à l'instant, à propos de Fréchette et Crémazie. Ce jugement sur les Laurentiennes de Sulte résume assez bien la situation: Il s'exhale de cette fraîche et douce invocation [...] un souffle de pur sentiment patriotique qui, à défaut même d'autres qualités, devrait désarmer la critique la plus malveillante (Le Monde, 26 février 1876).

Les œuvres canadiennes se sont-elles parfois vu adresser quelques reproches? Bien rarement, il faut le dire. Il y a certes Isidore Lebrun qui, dans la Revue encyclopédique (juin 1831), avait signalé les idées mal coordonnées, de même que le style heurté, incorrect et diffus de Bibaud (p. 530); mais, dans son Tableau statistique... il s'était montré plus généreux que dans son article de 1831 (cf. p. 270). Seul Lefaivre, en 1877, se permettra des jugements sévères, comme d'ailleurs la revue Polybiblion ${ }^{31}$.

\subsubsection{Conclusion pour la période 1830-1880}

S'il fallait caractériser la réception des œuvres canadiennes durant cette période, il faudrait parler d'un accueil par sympathie. D'abord curieux, puis étonné par la survivance de sa colonie perdue et de ses traits français, le critique cherchera toutes les caractéristiques qui composent l'image d'une France transatlantique: peinture fidèle du Canadien qui en fait un Français d'outre-mer, amour de la mère patrie, utilisation de la langue française, patriotisme et catholicisme. Ainsi s'élabore un appareillage propre, en quelque sorte, à apprivoiser la colonie perdue. Cette reconquête par le contenu entraîne une occultation des faiblesses et des vétustés formelles de la littérature canadienne, et ce biais explique l'impossibilité d'ériger le modèle français en canon littéraire: l'ordre du proverbe exige qu'avant de châtier, il faut d'abord aimer. De tous côtés, on est disposé à accueillir la littérature canadienne avec une batterie d'excuses:

[...] nous sommes bien plutôt porté à admirer les auteurs qui écrivent notre langue comme les poètes canadiens, qu'à protester 
contre quelques rares infractions au goût que l'on rencontre chez eux. En poésie comme en toute chose, il faut d'ailleurs tenir compte du temps et des circonstances. Or, les circonstances, nous ne saurions trop le dire, n'ont pas été jusqu'ici de nature à communiquer à la lyre canadienne cette délicatesse exquise et cette unité de ton que l'on retrouve chez certains de nos poètes. En revanche, les Canadiens rencontrent, sous le coup de la double inspiration du patriotisme et des scènes grandioses d'une nature incomparable, des accents admirables. (Edmond Farrenc, Le Constitutionnel, 31 mars 1874)

Voilà pourquoi les jugements négatifs, ou même partagés, cèdent devant les nombreuses appréciations généreuses de cette littérature. Celles-ci dépassent même parfois la mesure. I. Lebrun a tout de même dit, du recueil de Bibaud: des versificateurs, dans nos départements, publient des recueils de leurs poésies, qui, certes, sont inférieures à celles du poète canadien (Revue encyclopédique, juin 1831, p. 532). Charles Guérin, a-t-on déjà dit, est mieux qu'un bon livre; de Lourdoueix a qualifié le Canada reconquis par la France de livre remarquable. Ne ménageant rien, Marie Fontenay avait écrit, un mois plus tôt, que la littérature canadienne comptait plusieurs ouvres remarquables (Gazette de France, 27 février 1855). Dans la Tribune lyrique, l'auteur, dans une présentation des lettres canadiennes, choisit Fréchette en disant: Je n'en choisirai qu'un entre cent [...]! (Cité par P. de Cazes dans Le Monde, 17-18 avril 1876). Ce même de Cazes qualifiera Sulte d'excellent poète (Le Monde, 26 février 1876).

Pourquoi tant d'enthousiasme et si peu de discernement? Les jugements plus «éclairés» de Lefaivre et de Frary feront-ils école? On ne pourra répondre à ces questions qu'en examinant les années 1880-1900. Mais comme le ton semble changer vers 1880 , consacrons la deuxième partie de cette étude à Fréchette et à son "rival", Octave Crémazie.

\subsection{Le tournant des années 1880 : la réception instable des œuvres de Fréchette et de Crémazie.}

Nous avons posé, d'une part, l'hypothèse d'une première réception, située grosso modo entre 1830 et 1880 , et dont on sait maintenant les caractéristiques fondamentales. D'autre part, on aura peut-être observé que notre corpus s'arrêtait en 1878. Ce n'est pas un hasard.

En effet, à partir de cette date, et pour plusieurs années à venir, Louis Fréchette dominera la scène littéraire avec son recueil Pêle-mêle (1877), qu'il a bien pris soin de diffuser un peu partout, et avec la réception d'un prix Montyon, décerné par l'Académie française le 5 août 1880 pour les Fleurs boréales. Les oiseaux de neige. Mais Fréchette a un rival, du moins si l'on considère la critique française: Octave Crémazie. Il apparaît donc intéressant de suivre la réception critique accordée à ces deux poètes. Et cet intérêt est accru par le fait que les années 1880 amorcent une transformation de l'horizon $\mathrm{d}$ 'attente français. Dans cette deuxième section, nous devrions ainsi voir à la 
fois se consolider la réception par sympathie que nous connaissons, et apparaître les éléments qui composeront le second horizon (1880-1900). Qui considère-t-on, de Fréchette ou de Crémazie, comme le premier poète canadien? L'instabilité des normes de cette ère de transition fera en sorte que, jusque vers 1878, Crémazie aura au moins autant de notoriété que Fréchette; qu'entre 1878 et 1881 , Crémazie sera totalement confiné aux étagères de bibliothèque, pendant que Fréchette envahira les journaux; enfin, qu'après 1881, Fréchette perdra un peu d'altitude tandis que Crémazie sortira de l'antichambre de l'institution littéraire.

\section{Fréchette? Crémazie? Crémazie? Fréchette?}

En 1864, La Tribune lyrique, que cite Paul de Cazes, s'était montrée vaguement favorable à Mes loisirs de Fréchette, publié l'année précédente; et, ajoute de Cazes, pour une raison ou pour une autre, Mes loisirs ne reçurent pas généralement l'accueil auquel le jeune poète devait s'attendre (Le Monde, 17-18 avril 1876). De Cazes s'inquiète par ailleurs des écarts où pourrait l'entraîner parfois sa trop grande imagination de poète et de la vigueur parfois un peu rude de l'expression [...] (id.). Rien ne laisse pressentir l'année faste de 1880 pour le poète; bien au contraire, plusieurs lui préfèrent Crémazie. L'on reconnaît le souffle, le patriotisme de Fréchette, mais de Crémazie on avantage la muse plus robuste, les vibrations de sa lyre plus puissantes, et son inspiration plus uniforme et mieux soutenue $[\ldots](\mathrm{E}$. Farrenc, Le Constitutionnel, 31 mars 1874); un pas de plus, et Crémazie devient le représentant de la poésịe française en Amérique (id.). Lefaivre appuie Farrenc, qualifiant Crémazie du plus distingué membre de l'école nouvelle. En effet, si Fréchette représente le plus réputé des poètes canadiens (Conférence...1877, p. 28), ses conceptions n'ont peut-être pas beaucoup de force (id.). Plus généreux lui aussi pour Crémazie, Lefaivre conclut sur Fréchette qu'il fait des vers par dilettantisme (id.). Dans le Journal officiel de la République française, Raoul Frary passe carrément d'une laconique allusion aux pièces légères de Fréchette à l'heureux exemple de ce que le sentiment patriotique peut fournir d'inspirations élevées, citant un extrait du “Drapeau de Carillon» (dans la Revue de Montréal, janvier 1879, p. 10-11).

Crémazie jouit donc d'une notoriété égale, sinon supérieure à celle de Fréchette qui, il convient de noter, n'avait que deux recueils de jeunesse à son actif. Mais nous sommes en 1878; à partir de maintenant, l'auteur de Pêlemêle se voit commenté abondamment par la presse française. L'audience qu'on lui fait alors représente la provenance même des recueils: un livre qui nous vient de la France du nouveau-monde doit être le bienvenu dans la mère-patrie ${ }^{32}$; du Canada, de ce pays français par l'origine et par le coeur $[\ldots]$, il nous arrive un volume de poésie $\left[\ldots{ }^{33}\right.$. Pour les Fleurs boréales, la question est plus délicate: on sait que seuls les Français pouvaient concourir aux prix Montyon. Grâce à d'opportunes amitiés, en l'occurrence Xavier Marmier et le lauréat de l'année précédente, Prosper Blanchemain, Fréchette se présenta et l'Académie s'empressa de l'ouvrir [sa porte] à ce Français du nouveau monde (C. Doucet, Concours littéraires..., 1886, p. 172). Sans insister sur les mérites intrinsèques de l'ouvrage, et stipulant que le 
couronnement n'était pas une affaire de faveur, Doucet avoue pourtant que la fraternité suffisait pour que les Poésies Canadiennes fussent admises à concourir $[\ldots]$ (id., p. 173).

Tous les lieux communs qui ont scandé les cinquante dernières années se retrouvent dès lors dans l'appréciation des poèmes de Fréchette: amour de la mère-patrie, culte du français et de la francité, souci du vrai et du beau, catholicisme et patriotisme et, pour tout dire, hégémonie du contenu.

Ainsi, note L'Indicateur ( 14 juillet 1878), les vers de Fréchette révèlent un attachement profond pour la mère-patrie [...]. Et quelle pureté de langage!, s'exclame Théodore Vibert ${ }^{34}$ : cela tient sans doute à ce que Fréchette est Français de caeur ${ }^{35}$, ce qui lui permet de parler notre langue avec la facilité et la correction d'un Parisien de race ${ }^{36}$. Le critique français reflétera cet amour de la France en livrant des extraits de poèmes qui, pour l'immense majorité, feront état de l'amour du vieux sol qu'à bénir on s'obstine (vers de Fréchette lu par C. Doucet lors de la remise du prix). Dans la foulée du réalisme de reportage, les poèmes de Fréchette seront en outre goûtés parce que ses descriptions sont vraies (Le Parnasse, 15 novembre 1878 ), et que l'œuvre exalte avec un égal enthousiasme les bienfaits du catholicisme et les jouissances de la liberté (id.).

On apprécie donc d'autant plus la poésie de Fréchette que se dessine, derrière toute lecture, cet horizon de sympathie que nous connaissons déjà. Fréchette écrit bien, il ne s'éloigne pas de la réalité, et il est français de coeur; voilà bien des raisons pour que l'auteur de Pêle-mêle aît [sic] toutes nos sympathies, peut-on lire dans Le Parnasse (15 novembre 1878). Il s'ensuit que les comparaisons avec les auteurs français seront parfois avantageuses pour le Canadien, mais le plus souvent neutres, et jamais défavorables. Le Journal de Montmédy discernera un souffle parnassien (8 février 1877); la Gazette des Dimanches évoquera une parenté littéraire avec nos poètes de la grande période romantique ${ }^{37}$; sera relevée l'influence de Lamartine et de $\mathrm{Hugo}^{38}$. Toutes ces affinités évitent d'établir une véritable comparaison ou, le cas échéant, feront pencher la balance du côté de Fréchette, Musset dût-il en occuper l'autre plateau: Dans Réminiscor, il [Fréchette] égale Alfred de Musset par la grâce, mais il le surpasse par une perfection de versification à laquelle ne sut jamais arriver le délicieux poète (Le Propagateur du Var, février 1878). Sans réserves, L'Artiste clame: [...] Lamartine et Hugo n'ont pas mieux poétisé la langue française ${ }^{39}$. Ici, plus que jamais, ces comparaisons dénotent un passéisme qui convient bien aux journaux de province...

Comment le critique français s'y prendra-t-il pour exprimer cette lecture par sympathie, cette communication par le cœur? Se dessine-t-il une sorte de leitmotif qui en témoigne? La récurrence d'un mot, pour qualifier les vers de Fréchette, le mot charmant, semble tout à fait approprié et, mieux que tout autre par sa valeur et par l'usage abusif qui en est fait, il exprime cette médiation affective entre l'ouvre et le critique. Cette accumulation est trop abondante pour relever du hasard: un charmant désordre (L'Indicateur); des poésies [...] charmantes (Revue des Idées nouvelles); vos vers sont 
charmants (lettre de Jules Simon, dans L'Opinion publique, 7 novembre 1878); vos chants canadiens [...] sont pleins de charme (lettre de Gustave Droz, dans L'Opinion publique, 14 novembre 1878); on y respire [...] les charmes pénétrants de l'esprit (lettre de Prosper Blanchemain, dans L'Opinion publique, 26 décembre 1878); un charmant recueil de poésies (Le Parnasse, 15 novembre 1878); l'ouvrage en question est plein de charme (Gazette des Bouches du Rhône, 22 septembre 1877); un charmant recueil de poésies (Journal d'Elbouf, I ${ }^{\text {er }}$ janvier 1879), et nous en passons! Plutôt que d'une saisie rationnelle, le critique préfère une approche sentimentale, non exempte de condescendance, propre à cette réception dont nous avons fait état.

Durant les années, donc, qui vont de 1878 à 1881 , de Crémazie il n'est point question; Fréchette domine la scène tout entière et, répondant parfaitement aux attentes du lecteur français, il est honoré, élevé à la hauteur de poète national. Le mérite et la valeur de ses œuvres justifiaient-ils pareil hommage? N'est-ce pas parce que, en plus de chanter la fidélité à la France, il permettait à ce pays d'apposer un sceau tout français sur une œuvre canadienne? Il est en effet très difficile, dans la présentation qu'a faite Camille Doucet, de distinguer ce qui relève de l'hommage rendu en pareilles occasions à la littérature d'avec celui, souvent plus décisif, rendu à la fidélité envers la France ${ }^{40}$. Et sans doute sera-ce pour protester contre ce manque d'objectivité que Louis Dantin déplorera plus tard que l'Académie ait encouragé des illusions ridicules ${ }^{41}$.

Quoi qu'il en soit, à partir de 1881, l'œuvre de Crémazie commence à réapparaître. La mort en France du poète, deux ans plus tôt, y est-elle pour quelque chose? Dans Le Monde, le comte de Foucault parle de Crémazie comme de l'un des poètes canadiens les plus en vue [...], lui consacrant deux articles, et lui reconnaissant cette qualité si rare et si précieuse qui n'est dévolue qu'au vrai poète: l'invention ${ }^{42}$ (11 mars). Désormais, le nom de Fréchette n'apparaîtra plus seul: il devra s'accompagner de celui de Crémazie, car tous deux sont sacrés poètes ${ }^{43}$. Et, renversant les pôles, de Baudoncourt n'hésitera point à considérer Crémazie comme le poète national ${ }^{44}$. Mais il revient probablement à Gailly de Taurines, dans La Nation canadienne ${ }^{45}$, d'exprimer le mieux ce renversement: consacrant à peine une page à Fréchette, il commentera le "Drapeau de Carillon" et le "Vieux soldat canadien" pendant plus de cinq pages!

Comment expliquer cette baisse de popularité du lauréat de l'Académie? Certes, l'enthousiasme outré de son œuvre, dans les années 1877-1881, ne pouvait durer; tôt ou tard, la critique française devait relativiser ses jugements. Mais à la faveur de quels changements dans son système normatif? Voilà la question qu'il faut maintenant débattre dans une troisième partie de notre enquête, consacrée aux années 1880-1900. 


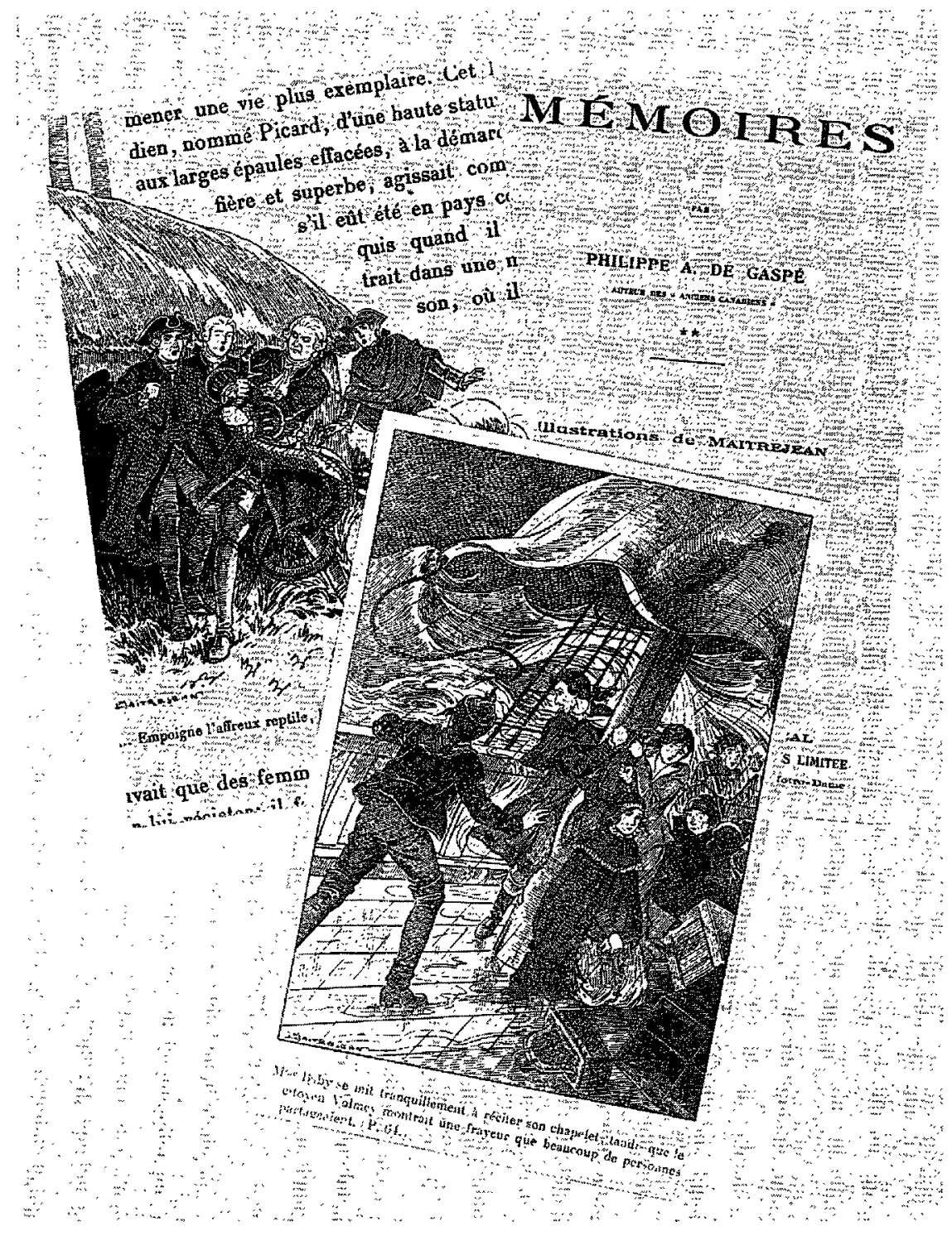




\subsection{La seconde réception des lettres canadiennes-françaises, 1880-1900}

\subsubsection{La transformation de l'horizon d'attente.}

Malgré ce que nous avons dit au sujet d'une transformation de l'horizon d'attente à partir de 1880 , on aurait évidemment tort de croire que les critiques français balaieront du revers de la main le système de références qui les a guidés depuis un demi-siècle dans leur appréciation de la littérature canadienne. Victor Du Bled éclaire un chemin pourtant déjà très fréquenté dans sa description, en 1885, de l'orientation de la littérature du Canada français:

Se retremper sans cesse dans l'étude du passé, ressusciter les glorieuses annales, recueillir avec un soin pieux ses légendes, s'identifier aussi avec le présent, peindre les moeurs, la vie sociale contemporaine, noter et nous traduire la majestueuse symphonie de la terre canadienne, $[\ldots]$ tel est, pour longtemps encore, le rôle, le devoir des écrivains canadiens [...] (Revue des Deux Mondes, p. 880)

On s'attendra donc à ce que le Canadien français montre les mêmes traits dans son œuvre - francité, simplicité, etc.--, auxquels on assortira de nouvelles coordonnées de lecture. Passons donc en revue l'ancien et le nouveau de ce second horizon d'attente.

\section{Persistance de l'ancien}

Dans son appréciation de la poésie de Crémazie, Foucault parle un langage connu, celui de l'amour de la France, amour sans bornes (Le Monde, 1881), de la présence des deux sentiments les plus nobles qui puissent animer un poète: le patriotisme et la foi (id.) Les mêmes vertus de simplicité, de vérité sont également au programme: Les romanciers canadiens, disons-le à leur honneur, ne sacrifient pas au goût malsain de tant de nos romanciers de ce côté de l'Océan $[\ldots]$ 46. Quoi de mieux que ce roman "honnête», selon le mot de Réveillaud, pour plaire à ceux auxquels le goût du jour, fût-il parnassien ou naturaliste, répugne:

Le caractère général de leur littérature est la simplicité, l'honnêteté, le respect du public et de soi... et le Saint-Laurent aura roulé bien des flots à la mer avant que l'école réaliste s'acclimate sur ses rives, et s'attache à révéler aux scandalisés, les plus infimes dessous des assomoirs et des lieux pires encore, qui doivent se cacher dans les quartiers de Montréal ou de Québec ${ }^{47}$.

N'insistons point sur cette panoplie de vertus trop familières, et portons plutôt nos regards vers les nouveaux étalons qui seront apposés sur la littérature canadienne. 


\section{L'irruption du nouveau}

Ainsi, quelque chose a changé depuis 1880 , qu'on pourrait caractériser par une attitude moins enthousiaste, plus critique de la part du lecteur français. Cette nouvelle lecture, qui confinera parfois à la sévérité, s'exercera sous les traits suivants.

\section{L'ère du soupçon}

L'Histoire de Garneau, on se le rappelle, a éveillé l'intérêt des Français pour la littérature canadienne; plus encore, des expressions de sympathie affluèrent de France, l'enthousiasme remplaça l'oubli [...] (Gailly de Taurines, la Nation canadienne, 1894, p. 260). Mais pareille sympathie ne pouvait durer, appelant un dépassement de la prise de contact initiale: Nous bornerons-nous, demande Du Bled, à quelques témoignages de sympathie littéraire et ne lui donnerons-nous pas des preuves plus solides de notre affection? (Revue des Deux Mondes, 15 février 1855, p. 881).

Or, cette affection prendra un visage singulier, celui, d'une part, de soumettre la littérature canadienne-française à un jugement beaucoup plus rigoureux, pour, d'autre part, la rendre dépendante des modèles français et de «l'attente du chef-d'cuvre». À ce prix le Canada français pourra-t-il devenir une "colonie intellectuelle» de la France. Voyons les composantes de cette critique plus sévère.

Dans une France qui, depuis 1880 , fait la vie dure au catholicisme, il va sans dire que le Québec ultramontain offre une grande consolation aux fidèles. Cependant, même à ce chapitre, il s'en trouve pour fustiger certains écrivains canadiens qu'on peut soupçonner de tiédeur religieuse. Cherchant querelle à Xavier Marmier pour sa trop grande sympathie pour le Canada, un article anonyme de la Revue littéraire lui reproche de se montrer trop indulgent pour certains auteurs canadiens dont la plume est aujourd'hui vendue à la Révolution, ce fléau de l'influence chrétienne et française ${ }^{48}$. Mais le contenu n'est pas seul à subir ces rigueurs; on n'est pas plus tolérant pour la langue. Dorénavant, le seul fait d'écrire en français ne suffit plus: l'auteur canadien doit témoigner de la maîtrise d'une langue correcte, policée même. Eugène Réveillaud refuse toute facilité en affirmant que la discipline littéraire doit être plus étroite encore au Canada qu'en France, si l'on veut y préserver la langue immortelle du "grand siècle» des envahissements et des empiétements de la langue voisine (Histoire du Canada..., 1884, p. 536). Un pas de plus, et cette langue, prenant Paris pour norme, perdra sa couleur locale; la langue de Louis Fréchette, dans sa Légende d'un peuple, note avantageusement de la Brière, est irréprochablement française, sans que le terroir s'y accuse par aucune trace fâcheuse ${ }^{49}$. Par une formule concise, de la Brière décrète: son encre vient certainement de Paris (id., p. 22).

De ces propos de censeurs commence à sourdre une attitude beaucoup moins magnanime qu'auparavant. Ce nouvel horizon d'attente gravitera autour de deux repères constants: le souci de la forme, et le modèle littéraire français. 


\section{Le souci de la forme}

Naguère, la vie était belle pour l'écrivain du Canada, qui n'avait qu'à défiler des scènes du pays, emmêlées de patriotisme pour la France, pour que le critique passe outre à une forme parfois bien chétive. Ces excuses n'ont maintenant plus cours: l'écrivain canadien devra, pour se plier aux exigences du lecteur français, faire preuve d'un souci et d'une maîtrise de la forme qu'on ne lui avait pas demandés encore. Après l'inspiration, l'élévation de la pensée, il faut le polissage formel:

Au reste, sans demander aux poètes canadiens d'avoir le fétichisme de la rime, d'attendre d'elle la grâce et le salut, on peut leur reprocher de n'avoir pas suffisamment étudié nos modernes: Laprade, Leconte de Lisle, Théodore de Banville, de ne pas comprendre assez que la poésie est non seulement un don du ciel, mais aussi une science et un art. (Du Bled, Revue des Deux Mondes, 1885, p. 878)

Il est vrai que les Canadiens ont peiné, en leurs débuts littéraires, et que le Parnasse leur était peu connu. De la Brière l'admet: Ils ont toujours eu la verve, l'élan, l'idée: ils ont conquis le savoir; la forme vient à son tour (Samedi-Revue, 1888, p. 23). Montrant une audace que peu de ses compatriotes auront, Rémy de Gourmont ira jusqu'à dire que, quant au choix des sujets, il est presque indifférent ${ }^{50}$.

Le souci de la forme est une chose; mais selon quel critère la juger? Car, pour élargir la question, il faut noter que jusqu'à présent le critique français ne disposait vraiment d'aucune norme locale autorisant un jugement sur la "qualité littéraire» de l'œuvre canadienne. On disposait d'une batterie d'attentes, certes, mais il manquait ce modèle, quasi platonicien, à partir duquel les appréciations pourraient être validées. Faut-il dire que nous touchons ici à une pression, voire à une sujétion qui a marqué la littérature du Québec jusque dans les années 1950 ? Il s'agit, bien sûr, du «modèle littéraire français".

\section{Le modèle littéraire français}

Voilà une question beaucoup plus délicate qu'il n'en paraît de prime abord. Nous le savons maintenant, de même qu'il serait faux de dire que la littérature canadienne s'est abritée sous le parapluie français dès ses débuts, de même serait-il réducteur de poser que le modèle littéraire français s'est affirmé tout de go, sans nuances et, aussi, sans contradictions.

Jusque dans les années 1880 , on n'avait mis en présence les écrivains canadiens et français que par une sorte de contiguité, épargnant les premiers de toute comparaison qui pût leur être favorable. Encore en 1881 , J. de Foucault dira, de Crémazie, que ses vers rappellent le Hugo des Odes et Ballades. Et il se permet en outre une comparaison fort subtile, qui n'éclabousse pas trop le poète canadien: il fait partie des littérateurs qui pourraient sans trop de désavantages, soutenir la comparaison avec les représentants actuels de notre école poétique (Le Monde, 11 mars 1881). Avec le même 
ménagement, tout en affirmant avec plus de force la nécessité du modèle français, Réveillaud écrira:

Cette littérature existe dès à présent au Canada; elle a tous ses organes; et quoiqu'elle doive longtemps encore, selon toute apparence, être tributaire de la France pour l'étude de nos inimitables classiques et le choix des plus parfaits modèles dans tous les genres littéraires, elle a déjà une vie propre et indépendante, une inspiration souvent originale et prime-sautière. (Histoire du Canada ..., 1884 , p. 542 )

Comme, en effet, longtemps encore, la littérature canadienne-française contemplera l'idéal français! Et comment ne pas relever l'impossibilité, pour une littérature, sinon d'imiter, du moins de s'accomplir par rapport aux inimitables classiques et aux plus parfaits modèles! Cet écart infranchissable grèvera longtemps les œuvres canadiennes-françaises, et l'on peut voir ici le point de départ de cet impossible achèvement ${ }^{51}$.

Longtemps, il a été possible d'entendre, suite à la parution d'un livre québécois important: «Tenons-nous enfin notre chef-d'ouvre? "Cette hantise de l'œuvre-maîtresse, on peut la situer, du point de vue français, après les années 1880 . L'attente du chef-d'œuvre, nous pouvons d'abord la trouver dans la Revue du monde catholique. Parlant du Papineau de Fréchette, R. Martin lance cette phrase où s'exprime bien la conjonction du modèle français et de l'œuvre parfaite: [...] est-il bien sûr que Papineau soit un chef-d'œuvre digne des grands maîtres français? 52 De Gourmont, quelques années plus tard, contribuera à l'obsession du chef-d'œuvre: après avoir, comme on le sait, accordé peu d'importance au choix du sujet pour "en tirer des chefsd'œuvre", il conclut: Qui sait? demain, peut-être, notre littérature d'outremer aura cette consécration (Les Canadiens de France. 1893, p. 211).

Position paradoxale que celle de cette littérature canadienne-française, censée produire des chefs-d'œuvre à l'ombre d'une littérature-mère dont les modèles sont inimitables! Et il y a plus: d'une part, le lecteur français aiguille l'écrivain canadien sur la voie de ses propres références, tout en hésitant lui-même, d'autre part, sur l'école littéraire à suivre. Ici, l'on invitera le Canadien français à tirer parti des modèles classiques (Réveillaud); là, on lui suggérera plutôt de se mettre à l'écoute des modernes, des parnassiens ( $\mathrm{Du}$ Bled); et, entre les deux, il se trouvera toujours quelqu'un (de Foucault) pour rapprocher, non sans plaisir, quelque écrivain canadien des romantiques français. Il est certain que la critique française ne constitue pas à cette époque un champ homogène, si bien que l'écrivain du Canada se retrouve à un carrefour fléché d'indications contradictoires. Qu'à cela ne tienne, on peut encore accroître la confusion, fondée cette fois sur cette frontière fragile qui sépare l'imitation du pastiche.

L'intenable de la situation apparaît maintenant au grand jour: s'inspirer du modèle français, sans donner dans la servilité et le pastiche. Ou, d'un autre point de vue: le choix du sujet étant depuis quelques années moins 
important, faire original à l'intérieur des sentiers battus. Comment s'étonner, alors, que l'on accuse de pastiche la littérature du Canada français tout entière? Un autre écueil sur lequel ils sombrent fréquemment, c'est la tendance au pastiche, le besoin d'imiter quelque écrivain français, d'exagérer ses qualités et ses défauts [...] (Du Bled, Revue des Deux Mondes, 1885, p. 877). C'est justement en 1885 que Sylva Clapin déplorait la perte d'originalité de la littérature canadienne-française (voir plus haut, note 8). Résultat de l'attraction grandissante de la France, cette dépossession sera aussi déplorée l'année suivante par Feyrol:

Malheureusement, le cachet d'originalité qui faisait le charme principal de la littérature canadienne tend à disparaitre chaque jour; ce changement est dû certainement à l'influence des productions de France; les écrivains du Canada ont subi la séduction, et ils cessent d'être eux-mêmes, pour devenir des copistes de nos auteurs à la mode 53 .

Comment sortir de l'impasse? Comment écrire sous l'égide des maîtres français et ne pas encourir le reproche d'avoir voulu imiter d'une façon quelque peu servile les auteurs français les plus en renom et d'exagérer leurs qualités et leurs défauts (de Baudoncourt, Histoire populaire..., 1888, p. 459)?

En vérité, ces tâtonnements ouvrent la voie à la théorie du "retard littéraire" de la littérature canadienne de Rossel: [...] elle est en retard d'un quart de siècle et plus sur celle de la France, car les idées et les formes littéraires font lentement le voyage de Québec à Paris («Les poètes français du Canada contemporain", Revue d'histoire littéraire de la France, p. 465; voir D. M. Hayne, «Les lettres canadiennes en France IV ", Revue de l'Université Laval, p. 507). La thèse de Rossel résout toutes les contradictions: la littérature canadienne prend le pouls de la France, mais le décalage temporel lui impose une discordance désormais systématisée. Disons donc que cette théorie de Rossel marque le terme d'une dizaine d'années d'hésitations normatives, et cristallise une position qui aura longue vie.

Le langage critique, soutenu par un nouvel horizon d'attente, s'est donc mué, depuis 1880 , en une influence prépondérante exercée par la mère-patrie, modulée surtout à travers une attitude critique plus sévère, une plus grande attention portée à la forme et à l'apposition du sceau français sur les œuvres canadiennes-françaises. Passons maintenant en revue les principales ouvres commentées.

\subsubsection{La réception des œuvres}

Il se trouvait, un peu avant 1880 , quelques Français pour affirmer l'existence d'une littérature canadienne-française. Sans changer d'opinion à ce sujet, on transformera plutôt le rapport de forces, dans les années subséquentes, ce qui fera écrire à Napoléon Legendre, qui s'inscrit lui-même en faux contre cette allégation, qu'on prétend que nous n'avons pas de littérature canadienne proprement dite, et que ce qui est écrit par nous fait partie des 


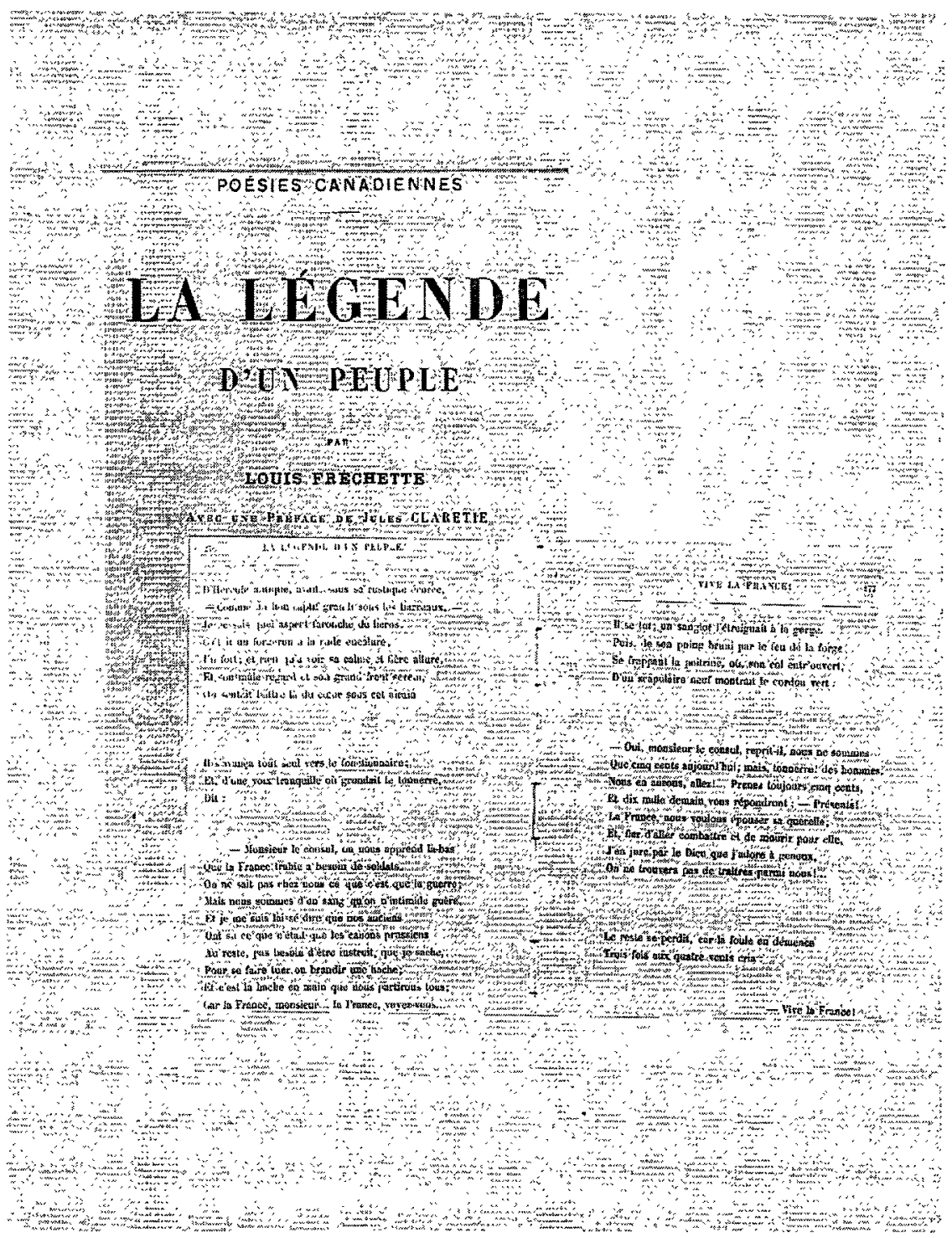


lettres françaises ${ }^{54}$. Quoi qu'il en soit, les ouvres seront reçues en France dans cette optique d'appartenance.

L'épisode de Louis Fréchette (et d'Octave Crémazie) nous a entraîné, pour ces deux auteurs, au delà des années 80 ; inutile de revenir ici sur leur réception. En revanche, Fréchette et Crémazie exclus, il reste bien peu d'œuvres ou d'auteurs qui aient été commentés avec une certaine ampleur, entre 1880 et 1900 . Du Bled, Réveillaud, de Baudoncourt, de la Brière se contentent d'une énumération où chaque auteur n'a droit qu'à quelques lignes, sauf exceptions rares. Même survol chez de Gourmont, encore que ce dernier soit le champion des erreurs (Barttre, Le Chevalier de Mornoc, l'abbé Tariguay, p. 20, 195 et 197). Le cas du Journal Paris-Canada et de la Revue des Deux Frances n'est guère plus resplendissant: peu d'articles littéraires sur le Canada français signés par des Français, sinon, des notices souvent brèves et portant peu à conséquence. Voyons pourtant cet esprit de géométrie d'un œil positif: cet intérêt accordé non pas à un auteur ou une ouvre, mais à l'ensemble des lettres canadiennes, annonce, malgré son peu de profondeur, l'envergure des entreprises de Rossel et, plus tard, de ab der Halden. Au delà des œuvres éparses, c'est la production d'ensemble du Canada français dont on veut maintenant parler.

Outre - nous le répétons - Fréchette et Crémazie que nous avons déjà traités, deux auteurs ont passablement fait parler d'eux: Barthe et l'abbé Casgrain. Dans un premier temps, nous caractérisons leur réception critique; puis, en deuxième lieu, nous examinerons de près l'étude de Rossel, pour montrer comment elle rassemble tous les courants de l'horizon d'attente de la fin du siècle, et quelle lecture des auteurs canadiens en résulte.

\section{"Le Canada reconquis par la France», de Barthe}

Ce livre a suscité beaucoup de commentaires, avant 1880 ; et on ne l'a pas oublié après cette date.

L'Histoire du Canada... d'Eugène Réveillaud est dédicacée à Jules Ferry, l'un des réalisateurs de la politique coloniale française qui a pour but de venger la défaite de 1870 . Faut-il s'étonner, dès lors, de l'intérêt tout particulier que Réveillaud accorde à Barthe? L'auteur trouve que cet ouvrage n'a pas eu, en 1855 , l'audience qu'il méritait. La cause? la politique coloniale mal avisée de l'époque (p. 516-517). Revenant sur le sujet un peu plus loin, Réveillaud dit que ce livre curieux [...] a mis en lumière, avec beaucoup de patriotisme, la nécessité de rétablir des relations suivies entre le Canada et son ancienne métropole (p. 539).

De Gourmont s'écarte de Réveillaud, cependant, en ce qui concerne l'accueil réservé au Canada reconquis par la France. Selon De Gourmont, ce livre a connu un grand succès, contrairement à l'opinion de Réveillaud. Mais là où les deux s'entendent à merveille, c'est sur la «lecture colonialiste" de l'œuvre. Il faut citer en entier ce morceau de bravoure: 
On a cru longtemps que nous étions incapables de toute initiative commerciale, mais le livre de $M$. Barttre [ sic], Le Canada reconquis par la France, a quelque peu modifié le sentiment à cet égard. À la suite de cet ouvrage, dont le retentissement fut considérable, des Français de France sont venus qui se sont demandé pourquoi l'ancienne mère Patrie ne tentait pas, en effet, une nouvelle et pacifique conquête de ce sol jadis et pendant si longtemps arrosé du sang de ses soldats. [...] Eh bien, en dépit des sceptiques, cette idée a germé, comme toute idée féconde et elle commence à porter des fruits. (Les Canadiens de France, 1893, p. 20 et 23)

Ces indications nous seront précieuses, lorsque nous aurons l'occasion de soulever certaines hypothèses concernant cette importante question de la politique coloniale française.

\section{«Les Canadiens de l'Ouest", de Joseph Tassé}

Sur la même lancée, et après s'êtrę contenté de quelques coups de pinceau rapides à propos d'autres ceuvres, Du Bled consacre une grande page aux Canadiens de l'Ouest, de Tassé. Plus que toute autre œuvre de prose, cet ouvrage attire l'attention de Du Bled, car $M$. Tassé a retrouvé une partie des titres de noblesse de la France dans le Nouveau-Monde, a contribué à dissiper ce préjugé banal qui interdit à notre race le don de la colonisation (Revue des Deux Mondes, 1885, p. 879).

A part, donc, les critiques sur Crémazie et Fréchette, de même que ces propos sur Barthe et Tassé, bien peu d'œuvres canadiennes-françaises se sont vu décerner plus qu'un trait de plume. De Bibaud fils, par exemple, Réveillaud écrira qu'il est souvent touffu et broussailleux comme son père, mais vif, original et d'une érudition aussi riche que variée [...] (Histoire du Canada..., 1884, p. 539); quelques lignes plus haut, on avait pu lire que l'Histoire de Garneau, avec des inégalités et des incorrections de style, renferme des beautés de premier ordre [...] (id.,). On aurait pu donner, à titre indicatif, les quelques mots que Martin a accordés à Routhier, pour $\bar{A}$ travers l'Europe (Revue du monde catholique, 1882, p. 110), ou que Foucault a consacrés aux Laurentiennes de Sulte (Le Monde, 25 mars 1881).

Prenons garde, néanmoins, d'en conclure à l'effacement de l'intérêt pour la littérature canadienne-française; voyons-là plutôt un effort du critique pour essayer d'embrasser l'ensemble des œuvres, présageant en quelque sorte les études à venir. Voilà pourquoi nous attacherons maintenant une importance toute particulière aux travaux de Rossel, qui conjuguent l'horizon d'attente et la réception de la fin du XIX' siècle et, par là, fermeront cette recherche. 


\section{Les études de Rossel: un aboutissement}

Nous avons déjà parlé des deux études de Virgile Rossel ${ }^{55}$, pour faire remarquer que, bien qu'il s'agisse d'un critique suisse, l'une de ses deux études est parue en France. Mais il y a plus: toute sa critique de la littérature canadienne-française s'est faite du point de vue de la France, ce qui donne à lire, entre autres, que le Canada français est une petite France du Canada (1895, p. 282). Aussi le considérons-nous parmi la panoplie des critiques français: d'esprit, Rossel l'est assurément.

Rossel signale la francité jalousement gardée des Canadiens français, et leur amour de la mère-patrie, pour laquelle il sont une passion exclusive (1894, p. 464). Muni de ces prémisses, il se pose face à la littérature canadienne-française de manière similaire à celle de la critique des années 1830-1880. Mais là où il participe de la tendance des vingt dernières années de son siècle, c'est lorsqu'il insiste sur l'isolement du Canada français, la nécessité de prendre la France pour modèle (d'où sa notion de retard), et les faiblesses de la forme des œuvres canadiennes.

Rossel avance prudemment sa «théorie du retard littéraire». Remarquant tout d'abord que le Canada est un coin de France du XVII siècle, perdu au-delà des mers, mais un coin de province française que le rayonnement de Paris n'atteindrait pas $(1894$, p. 465$)$, il doit déduire que les lettres canadiennes représentent une branche plus ou moins parasite, et une branche tardive perdue dans le feuillage (1895, p. 305). Rossel classera ensuite les écrivains selon leur position sur l'échelle de ce retard; il en sera de même pour les genres, comme pour le roman par exemple, qui manifeste un retard considérable, n'ayant même pas fait les conquêtes du romantisme français et, encore moins, du réalisme (cf. 1895, p. 332).

Rossel craint beaucoup, à l'instar de Du Bled, la société d'adulation mutuelle (1894, p. 477), ou encore l'oreiller de l'admiration mutuelle (1895, p. 307). Cette méfiance s'explique aisément: la manie d'adoration ou d'admiration mutuelle qui sévit avec une intensité toute particulière dans les milieux provinciaux [...] signifie le triomphe de l'à-peu-près en littérature (1895, p. 293). Une seule main tendue peut tirer les lettres canadiennes de cette ornière: les modèles littéraires de la mère-patrie. Il faudra, si les écrivains du Canada veulent éviter la facilité, qu'ils étudient, d'un oeil moins prévenu, d'un esprit plus libre de préjugés, le mouvement littéraire de la mère-patrie (1894, p. 465).

Les lettres canadiennes sont donc condamnées à être en retard, et leur étalon littéraire se trouvera en France. Leur inspiration, leurs sujets seront évidemment canadiens et, de ce côté, Rossel continue d'approuver les écrivains: leurs livres reposent sur un fonds solide de saines qualités et de fortes vertus nationales (1895, p. 298 ; aussi, 1894, p. 466). Mais, sur le plan de la forme, l'écrivain canadien exhibe toute sa faiblesse: C'est essentiellement du côté de la forme que les auteurs canadiens devront porter leur attention (1894, p. 465). Là surtout se montrent leurs carences et leur retard: Leur 
prosodie est à peu près celle des derniers classiques, et leur forme, plus frustre celle-ci, plus négligée celle-là (1894, p. 466). En fonction de ces normes et de ces attentes, Rossel pose ce jugement sur la poésie, qu'il reprendra, mutatis mutandis, pour toute la littérature canadienne-française:

\begin{abstract}
Le français de ses livres n'est point l'"écriture artiste», mais il rappelle, sinon par sa pureté, du moins par sa sobre et franche vigueur, celui de nos classiques. Son esthétique, surannée je l'accorde, et superficielle, a la bonne saveur et l'ingénuité des choses très simples. Sa conception du monde et de la vie est peut-être naïve; mais elle plaît par sa sincérité, elle est faite d'honnêteté et de foi. Et il n'est pas de source plus noble d'inspiration que celles où elle puise, le culte des antiques vertus, la religion des souvenirs sacrés. $(1894$, p. 465)
\end{abstract}

Tout au long de cette recherche, nous avons croisé les noms de Garneau, Chauveau, Fréchette, Crémazie, etc. Rossel les reprend tous, accompagnés de plusieurs autres. Voyons les principaux, à titre exemplaire de l'application de ce système normatif.

Cinquante ans plus tard, le jugement sur Garneau demeure favorable, malgré quelques faiblesses de style: son Histoire est [...] mieux qu'un livre: un acte (1895, p. 315). Tassé, dont les Canadiens de l'Ouest avaient attiré l'attention, mérite ici une longue page, ce qui n'a pas été le cas pour Barthe, bien qu'il ait replacé sa patrie dans la sphère de rayonnement de la civilisation française (1895, p. 319). Toujours au chapitre de la prose, le roman Charles Guérin, malgré un fond valable, apparaît filiforme sur le plan de sa trame: ce livre se fût signalé si la langue en avait été plus châtiée, la composition plus serrée, l'intrigue plus neuve (1895, p. 334). Comme nous nous sommes éloignés de l'enthousiasme de Marie Fontenay! Pourrait-il en être autrement dans un pays où la littérature se fait volontiers calme et terne $(1895, \mathrm{p} .336)$ ? Bien souvent, c'est le mot bluettes qu'on accolera aux ouvres du Canada français.

Heureusement, il y a la poésie... Mais ce n'est pas Lemay qui s'y taillera une place, car il manque d'haleine et d'envergure (1894, p. 468), en dépit de sa simplicité et de son charme. Les deux seuls poètes qui méritent une escale sont en vérité Crémazie et Fréchette.

Crémazie, dit Rossel, a de l'élévation, de l'inspiration, de la profondeur; plus encore, on lui découvre même un coin de génie (1895, p. 347). C'est dans sa "Promenade des trois morts» qu'il atteint sa plénitude, fiction grandiose malgré une forme insuffisamment travaillée et des sentiments qui auraient pu s'approcher davantage du catholicisme (1894, p. 475). Voilà identifié, d'ailleurs, le problème de Crémazie qui, poète national et barde canadien (1894, p. 473), demeure trop provincial, hésite entre le classicisme et le romantisme, et n'ose pas franchement se poser en écrivain français (1894, p. 471). 
Telles seront, en revanche, aux yeux de Rossel, les qualités principales de Fréchette, qui expliquent le léger avantage accordé à l'auteur de la Légende d'un peuple: s'il n'a pas la profondeur de Crémazie, il se montre plus égal et, surtout, plus moderne. Il a assimilé la nouvelle poésie française et, moins en retard, sa versification a beaucoup gagné $(1894$, p. 466) à se tenir au courant des mouvements littéraires de la France. Il est assez étonnant que Rossel ne fasse que saluer d'une phrase l'attribution du prix Montyon, à moins qu'il y voie là la politesse de quelques lauriers académiques (1895, p. 306), formulation qui sied tout à fait à l'interprétation que nous avons donnée de ce prix. Rossel s'attarde plutôt à la Légende, pour ses aspects robustes, sonores et. surtout, français, encore qu'il s'y manifeste quelques faiblesses de forme et que la langue trahisse un écrivain qui n'a pas été formé en France (1894, p. 484).

Rossel correspond à un fil d'arrivée, mais aussi de départ. Il a fallu quelque soixante années de critique pour en arriver à former ce système global d'appréciation littéraire; et, bien que pareil jugement déborde les limites que nous nous sommes données, sans doute nous permettra-t-on d'ajouter que ce nouveau point de vue marquera longtemps les lettres canadiennes-françaises.

\subsubsection{Conclusion pour la période 1880-1900}

Nous venons de clore à la fois la troisième et dernière partie de cette recherche. Concluons donc sur celle-ci, avant d'embrasser la recherche en entier.

En ce qui concerne tout d'abord la période qui couvre les années 1880 1900, la sévérité du lecteur français n'a pas manqué d'apparaître, mettant un terme à l'ère de fraternisation qui avait précédé. On préfère analyser, discuter, évaluer l'œuvre. L'époque des jugements généreux sera ainsi révolue et remplacée par des jugements de ce genre: Tout en admettant que cette petite troupe [des hommes de lettres canadiens] ait besoin de former une société d'admiration mutuelle, on demeure parfois confondus des complimens hyperboliques qu'elle décerne pour de biens chétives productions (Du Bled, Revue des Deux Mondes, 1885, p. 878; voir aussi Gailly de Taurines, La Nation canadienne, 1894, p. 278-279). L'exemple de Charles Guérin est peutêtre le plus éloquent de ce passage de l'engouement à la tiédeur. Cette époque voit également s'imposer les modèles français, encore que les critiques ne s'entendent pas sur l'école à suivre; aussi est-ce, comme par voie de conséquence, le début de cette longue période du chef-d'œuvre attendu et, jusqu'à un certain point, irréalisable. Citons le cas de Louis Fréchette: plus «moderne", plus conforme aux canons français, il verra sa popularité se consolider vers 1880 , en même temps que celle de Crémazie. Sans connaître vraiment de bas, la popularité de l'auteur de Mes Loisirs oscillera entre 1864 et 1900, les années 1880 et 1890 marquant les sommets.

Bref, 1880-1900 sera «l'ère de la forme»: le contenu seul des œuvres ne suffit plus comme passeport pour entrer dans la littérature, le travail du texte, la facture devant également s'imposer. Ainsi plusieurs œuvres ne franchiront-elles plus la frontière! 


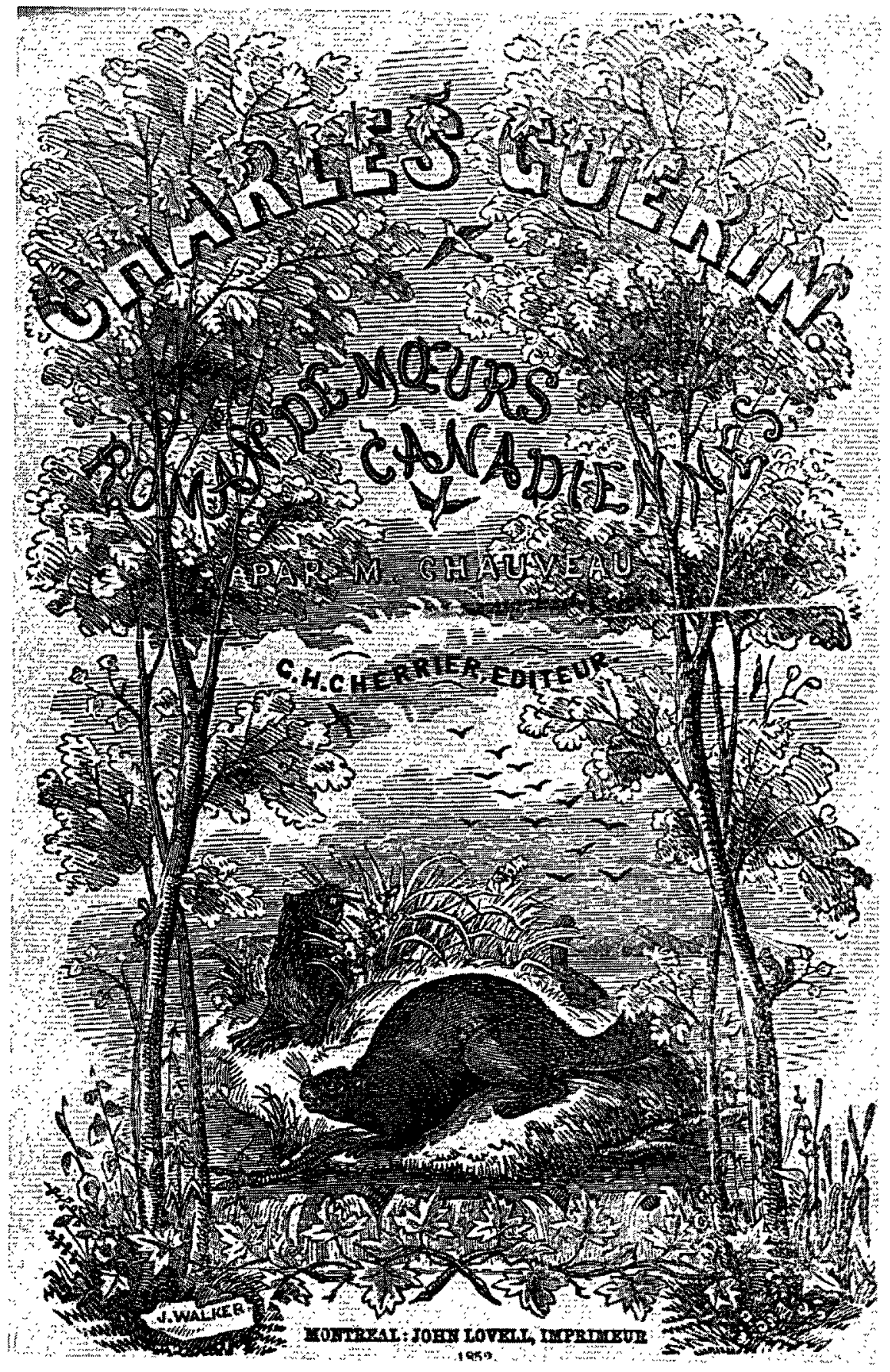




\section{Conclusion générale: remarques sur le changement d'horizon et de réception.}

Venons-en maintenant à notre conclusion d'ensemble. Ce serait simplement nous répéter que de reprendre tout ce mouvement d'horizon d'attente des années 1830-1900, scindé en deux par les années 1880 , frontière entre la sympathie et la critique, le fond et la forme, la canadianité et les modèles français. Interrogeons-nous plutôt sur ces changements d'horizon et de réception critique: pourquoi pareilles mutations? pourquoi en 1880 ?

La question mérite d'être abordée sur plusieurs fronts. Si l'on s'en tient à Jauss, il convient de dire que l'apparition d'œuvres nouvelles, bouleversant à des degrés divers l'horizon qui les précède, instaure une cinétique du changement qui anime le mouvement de l'histoire littéraire. Les œuvres «originales", nouvelles, sont revêtues d'un pouvoir tout à fait spécial. Mais la question se complique pour le cas que nous étudions: où se situera l'original, la nouveauté pour le lecteur français qui a passé à travers le romantisme, le réalisme, le parnasse et le symbolisme? Certes, le contenu canadien, de prime abord, l'étonnera; mais celui-ci aura vite fait de s'instituer en déjà-vu; plus encore, il sera rejeté vers 1880 . Il est d'ailleurs significatif que le critère d'originalité soit fort peu employé par le critique français: nous n'avons pu relever, dans cet ordre d'idées, que les indications de Lefaivre, qui trouve que la muse canadienne est plus à son aise, plus originale dans le poème comique et dans la chanson (Conférences... , 1874, p. 16), de Frary, pour qui la biographie semble un genre particulièrement original (Journal officiel de la République française, dans la Revue de Montréal, février 1879, p. 102), et de Rossel jugeant "La promenade des trois morts" comme une œuvre tout à fait à part. Mais ce n'est pas de ce côté qu'on réussira à expliquer la transformation d'horizon. Il faut plutôt se porter vers des causes externes.

Armand Yon ne procède pas autrement pour expliquer le changement d'attitude qu'il établit lui aussi vers 1880 . Le sens de la critique évolue rapidement, dit-il, dans la dernière moitié $\mathrm{du} \mathrm{XIX}^{\mathrm{e}}$ siècle. La psychologie et les disciplines auxiliaires se développent et sont appliquées à l'étude des peuples et de leur histoire (le Canada français vu de France, p. 69). A ce positivisme s'ajoute la polarisation religieuse: à l'ultramontanisme au Canada français correspondent deux courants de pensée français, le catholicisme et le «laïcisme", ce dernier devenant de plus en plus visible à partir de 1880. L'accueil fait au Canada français après cette date ne pourra plus être homogène, et la sympathie passera plutôt du côté de la droite.

Mais il est une autre cause externe que Yon omet de signaler, et dont la présence court dans toute la critique française: la politique coloniale de la France, que nous ne pouvons qu'évoquer pour finir.

Cette connexion possible entre la mutation d'horizon et la politique coloniale de la France n'est pas gratuite: elle nous est suggérée à la fois par les textes que nous avons étudiés, et par les événements qui ont marqué cette période. Voyons d'abord les événements. 
On sait que les années 1870 sont pénibles pour la France, particulièrement à cause de la perte de l'Alsace et de la Lorraine. De nombreuses personnalités françaises animeront l'idée d'impérialisme après ce revers, faisant naître le thème de la mission civilisatrice; et l'âge d'or de la colonisation aura lieu durant les deux dernières décennies du $X I X^{e}$ siècle et le début du $X X^{e}$ siècle $^{56}$. Or, c'est précisément à cette époque que se transforme le système d'attente français sur le plan littéraire et que, surtout, les allusions coloniales abondent dans les textes que nous avons déjà cités.

Comme le dit clairement Réveillaud: Aux Français, il reste l'Afrique du Nord et le Canada (Histoire du Canada, 1884, p. 511). Quels avantages présenterait la reconquête de la colonie perdue! La France doit ainsi faire du Canada français une colonie intellectuelle, selon l'expression employée par Du Bled et de Baudoncourt. Et le domaine littéraire représente un terrain magnifique à cet effet.

Mais ce ne sont là qu'hypothèses, qui ouvrent d'autres voies de recherches, celles-là mêmes des velléités colonisatrices de la France envers le Québec de la fin du siècle, soutenues par l'émigration et les échanges commerciaux. N'allons pas plus loin dans cette direction pour l'instant, et souhaitons simplement avoir fait apparaître clairement les changements d'horizon et de réception des lettres canadiennes-françaises dans une période où l'on a, trop souvent, considéré l'état de la question de manière statique et homogène. Le XIX ${ }^{e}$ siècle nous est en effet apparu plus complexe qu'on aurait pu le croire: la France ne s'est pas saisie d'un seul coup de la littérature canadienne-française, et son hégémonie normative a pris plus d'un demi-siècle à se constituer. Elle en prendra davantage à se désagréger.

1. Il s'agit de la relation du deuxième voyage de Jacques Cartier, Brief récit, \& succinte narration de la navigation faicte es ysles de Canada, Hochelaga \& Saguenay \& autres, avec particulières meurs, langaige \& ceremonies des habitans d'icelles: fort delectable à veoir, édité en 1545 par Ponce Roffet, dit Faucheur, et Antoine Le Clerc, frères, à Paris (in 8 de six cahiers, 48 feuillets en tout). Voir à ce sujet A. Leo Leymarie (Membre correspondant de l'Institut Canadien), Le Premier ouvrage français sur le Canada (1545), (extrait des procès-verbaux et mémoires du Congrès international des bibliothécaires et des bibliophiles, Paris, 1923), Paris, Jouve et Cie, 1925, 8 p.

2. David M. Hayne, «Les lettres canadiennes en France», 1 (1830-1860), La Revue de I'Université Laval, XV, no 3, novembre 1960, p. 222.

3. Hans-Robert Jauss, Pour une esthéthique de la réception. Paris, Gallimard, 1978, p. 49 ( 307 p.).

4. Virgile Rossel, "Les poètes français du Canada contemporain", Revue d'histoire littéraire de la France, 1, 1894, p. 464-485; cet article sera ensuite fondu dans un ensemble plus vaste, le «Livre troisième» sur les Canadiens français (p. 281-301) et la littérature canadienne (p. 302-353), dans l'Histoire de la littérature française hors de France, Lausanne, Payot, 1895, 531 p. Considérons que nous sommes cependant dans 
une situation limitrophe puisque Rossel, bien qu'il ait publié d'abord en France, est un juriste, littérateur, historien et critique suisse. Surtout, donc, de Charles ab der Halden, Etudes de littérature canadienne-française et Nouvelles études de littérature canadienne-française, Paris, F.-R. de Rudeval, 1904 et 1907. Son entreprise de critique de littérature canadienne-française remonte cependant, on le sait, aux années 1899-1900.

5. David M. Hayne, "Les lettres canadiennes en France», La Revue de l'Université Laval, XV, no 3, novembre 1960 , p. 222-230; no 4, décembre 1960, p. 328-333; no 5, janvier 1961, p. 420-426; no 6, février 1961, p. 507-514. David M. Hayne, compiler, «Preliminary Bibliography of the Literary Relations between Quebec and the Francophone World", Revue canadienne de littérature comparée, printemps 1979, p. 206217. Armand Yon, le Canada français vu de France (1830-1914), Québec, Les Presses de l'Université Laval, coll. "Vie des Lettres québécoises», no 15, 1975, 237 p. Gérard Tougas, la Littérature canadienne-française, Paris, P. U.F., 5e édition, 1974, 270 p. (particulièrement le chapitre VI, "La littérature canadienne dans ses rapports avec la France et sa culture", p. 235-254).

6. Gustave Lanctot, l'Oeuvre de la France en Amérique du Nord. Bibliographie sélective et critique, Montréal. Fides, 1951, 187 p.

7. S'il est une autre question complexe, dont les limites de cette étude ne permettent pas un examen approfondi, c'est bien la position des organes de la presse française dans l'articulation du champ idéologique, particulièrement durant les années 1875-1900. Les études que nous avons consultées à ce sujet, particulièrement l'Histoire générale de la presse française (sous la dir. de Claude Bellanger, Jacques Godechot. Pierre Guiral et Fernand Tarrou, t. 11, 1815 à 1871, t. 111, 1871 à 1940, 1969 et 1972), répartissent la presse en deux classes, conservatrice et républicaine. Or, en vertu de cette division, la vaste majorité des journaux et des revues que nous avons consultés se range clairement sous l'étiquette conservatrice, à l'exception du journal Le Temps; nombreux seront les périodiques légitimistes (L'Union, la Gazette de France, etc.) ou catholiques (L'Univers, Le Monde, Le Correspondant, etc.) qui se pencheront sur la littérature canadienne. Bref, si le champ intellectuel français est loin d'être unifié à cette époque, c'est un regroupement assez homogène, de droite qui, cependant, s'intéresse à la littérature française du Canada.

8. Les études parues sur les relations entre la France et le Canada français au XIXe siècle pourraient faire l'objet d'un chapitre entier; on peut les diviser en deux catégories. selon qu'elles traitent des relations culturelles ou littéraires. Sur le plan des relations culturelles signalons: Maurice Guénard-Hodent, la Tradition renouée. Les relations entre la France et le Canada depuis soixante années, Paris, éd. de Paris-Canada, 1930, 48 p.; Claude Galarneau, "Les échanges culturels franco-canadiens depuis 1763", Recherches et débats, "Le Canada français aujourd'hui et demain", no 34, mars 1961, p. 68-78; Jean Ménard, Xavier Marmier et le Canada, avec des documents inédits. Relations franco-canadiennes au XIXe siècle, Québec, Presses de l'Université Laval, "Vie des lettres canadiennes», no 4, 1967,211 p. L'étude la plus pertinente, pour notre perspective, est cependant celle d'Armand Yon, le Canada français vu de France (1830-1914), Québec, Presses de l'Université Laval, «Vie des lettres québécoises", no 15, 1975.237 p. En effet, l'auteur pose deux époques, l'une du «sentiment", l'autre de la "critique", qui sont près de nos propres conclusions. Sur le plan des rapports littéraires, Yon (p. 138-139 par exemple) se départit des nuances qui l'ont bien servi au plan culturel. En ce qui a trait aux relations littéraires proprement dites, voir, de David M. Hayne, "Preliminary Bibliography of the Literary Relations between Québec and the Francophone World", Revue canadienne de littérature comparée, printemps 1979, p. 215-216. Il convient d'y ajouter: Sylva Clapin, la France transatlantique. Le Canada, Paris, Plon. Nourrit et Cie, 1885, 263 p.: Alphonse Lusignan, "Nos premiers rapports littéraires avec la France", Nouvelles soirées canadiennes, V, 1866, p. 443-446; Louise Beaudoin, les Relations France-Québec: deux époques, 1855-1910, 1960-1972, Mémoire de D.E.S., Université Laval, 1974. La série d'articles 
de David Hayne dans La Revue de l'Université Laval constitue la meilleure mise au point sur le sujet (cf. note 5). Pour la période 1945-1975, on consultera avec profit le livre de Jacqueline Gerols, le Roman québécois en France, Montréal, HMH, Cahiers du Québec, 1984, 359 p.

9. Pierre de Boisdeffre, «Où va le roman? ». Recherches et Débats, «Le Canada français aujourd'hui et demain", no 34, mars 1961, p. 169.

10. Hans-Georg Gadamer, le Problème de la conscience historique, Paris, BéatriceNauwelaerts, 1963, p. 72 ( 89 p.).

11. Voir à ce sujet Terry Eagleton, Literary Theory. An Introduction, Minneapolis, University of Minnesota Press, 1983, p. 84 (244 p.).

12. Encore faudrait-il nuancer: la théorie de la réception s'est transformée, affirme Jauss, en théorie de la communication littéraire, c'est-à-dire en un rapport dialogique où la partie réceptrice et la partie émettrice sont également impliquées. "Au sujet d'une nouvelle défense et illustration de l'expérience esthétique», entretien de Charles Grivel avec Hans-Robert Jauss, Revue des sciences humaines, no 177, 1980-1, p. 8 et 9 (p. 7-21).

13. Nous avons mis à contribution, ici, plusieurs renseignements tirés de Réception critique des textes littéraires québécois, études présentées et rassemblées par Richard Giguère, Département des études françaises, Université de Sherbrooke, 1982, $203 \mathrm{p}$.

14. A. de Puibusque, «De la littérature française au Canada. Charles Guérin, roman de mœurs, par M. Pierre Chauveau (de Québec)", L'Union, 27 juillet 1855. Les deux autres articles sont parus le 29 et 30 juillet. Il est temps de dire que, vu le grand nombre de références à venir, nous donnerons, la première fois, une description bibliographique complète pour ensuite l'intégrer, lors des citations suivantes, à notre propre texte.

15. L.-A. Olivier, Répertoire national, t. 111, 1854, p. 235, cité dans L'Union du 30 juillet.

16. Albert-Alexis Lefaivre, Conférence sur la littérature canadienne, Versailles, Bernard, 1877. p. 19-20 (61 p.).

17. L'article est ainsi signé. Il s'agit sans doute d'Edmond Farrenc, l'auteur d'un Coup d'ceil général sur le Canada. Géographie, statistique, politique, finances, banques, productions, commerce, Extrait du Journal des Économistes, septembre 1874, Paris, Guillaumin et Cie, $1874,20 \mathrm{p}$.

18. E. F., «L'histoire, la poésie et le roman français-canadien", Le Constitutionnel, 5 mars 1874, reproduit dans le Journal de l'instruction publique, avril 1874, p. 57.

19. J. Varech, Revue des idées nouvelles, octobre 1878 , page couverture 3, reproduit dans L'Opinion publique, "Un poète canadien apprécié en France», 7 novembre 1878, p. 530 .

20. Camille Doucet. Concours littéraires. Rapports annuels 1875-1885, Paris, C. Lévy, 1886, p. 172 (scéance publique du 5 août 1880; sur Fréchette, p. 171-173); voir l'article du Figaro, 6 août 1880.

21. J.-J. Ampère, Promenade en Amérique; Ẽtats-Unis-Cuba-Mexique, Paris, Lévy, I861, t. I, p. 130 (421 p.)

22. Laurentie, L'Union, 26 août 1855 . Un premier article du même auteur avait paru le 21 août.

23. Isidore Lebrun, Tableau statistique et politique des deux Canada, Paris, Treuttel et Würtz, $1833,540 \mathrm{p}$.

24. Isidore Lebrun, Revue encyclopédique, t. 50, juin 1831, p. 530 (p. 529-532).

25. Th. Pavie, "Les Français du Canada», Revue des Deux Mondes, 15 juillet 1853, p 280 (p. 278-304). Il faut pourtant tenir compte du sens du mot à l'époque.

26. Marie Fontenay, «Le Canada. Lettre à M. de LaRochefoucauld, duc de Doudeauville", Gazette de France, 27 février 1855. Cette "lettre" sera reprise dans son volume, signé $\mathrm{M}^{\text {me }}$ Manoël de Grandfort, L'Autre monde, Paris, Librairie nouvelle, $2^{\mathrm{e}}$ éd., 1857,273 p. 
27. H. de Lourdoueix, "La France transatlantique», Gazette de France, 16 mars 1855.

28. Paul de Cazes est le signataire d'une série d'articles dans Le Monde: «Le Canada sous la domination anglaise", 31 janvier 1876; "La littérature française au Canada. Benjamin Sulte», 26 février 1876; «La littérature au Canada. II: Louis-Honoré Fréchette», 17-18 avril 1876; présentation, dans la section "Feuilleton", de Jean Rivard, le défricheur canadien. Scènes de la vie réelle, 6-7 août 1877; “Oscar Dunn", 12 octobre 1877; "Les Canadiens de l'Ouest, par Joseph Tassé», 30 octobre 1878.

29. Raoul Frary, «Le Canada français et sa littérature», Journal officiel de la République française, 3 octobre et 6 novembre 1878, parus dans la Revue de Montréal, novembredécembre 1878 (p. 607-614), janvier 1879 (p. 6-12), février 1879 (p. 101-108).

30. A.-A. Lefaivre, Conférences sur le Canada français faites à la Société des sciences morales le 3 juillet et le 17 août, Versailles, Bernard, 1874, p. 17 (60 p.).

31. Il serait long, et inutile, de faire état de la réception de toutes les cuvres mineures. Donnons cependant quelques indications bibliographiques, selon les auteurs critiqués et, le cas échéant, les ceuvres. Arthur Buies: A. Lefaivre, Conférence..., 1877, p. 46 sq.; abbé Casgrain: P. de Cazes, Le Monde, 11-12 septembre 1876; Henri-Émile Chevalier: M. Fontenay, Gazette de France, 1855; Oscar Dunn: P. de Cazes, Le Monde, 12 octobre 1877; L.-J.-C. Fiset: A.-A. Lefaivre, Conférence..., 1877, p. 15; Pamphile Le May: E. Farrenc, Le Constitutionnel, 31 mars 1874 (sur L'Église des Hurons), Polybiblion, novembre 1876 (sur Les Vengeances), A. Lefaivre, Conférence..., 1877, p. 25, Polybiblion, novembre 1879 (sur Une gerbe); Joseph Lenoir: E. Farrenc, Le Constitutionnel, 31 mars 1874 ; Joseph Marmette: E. Farrenc, Le Constitutionnel, 5 mars 1874 , R. Frary, Journal officiel de la République française, 3 octobre et 6 novembre 1878 . Ce sont là les principaux; celui qui a reçu le plus de jugements sévères a été $P$. Le May.

32. Gazette des Bouches du Rhône, 22 septembre 1877, dans L'Opinion publique, 6 février 1879.

33. L'Indicateur, 14 juillet 1878, dans L'Opinion publique, 24 octobre 1878.

34. Le Propagateur du Var, février 1878, p. 50.

35. Le Parnasse, 15 novembre 1878, dans L'Opinion publique, 2 janvier 1879.

36. Le Figaro, dans L'Opinion publique, 2 septembre 1880.

37. Gazette des Dimanches, 30 septembre 1877, dans L'Opinion publique, 2 janvier 1879.

38. Revue des Deux Mondes, ler octobre 1877, dans L'Opinion publique, 2 janvier 1879; voir aussi Le Temps, 7 août 1880: «M. Fréchette a parfois des accents à la Hugo $[\ldots] . "$

39. L'Artiste, septembre 1878, dans L'Opinion publique, 6 février 1879.

40. Gérard Tougas, la Littérature canadienne-française, p. 241.

41. Louis Dantin, Gloses critiques, Montréal, A. Lévesque, 1931, p. 71, dans Tougas, la Littérature-canadienne française, p. 241-242.

42. Comte J. de Foucault, "Un poète canadien", 11 mars et 13 mars 1881 (la citation se trouve dans la livraison du 11 mars).

43. Victor Du Bled, "La vie politique, sociale et littéraire au Canada (1840-1884)", Revue des Deux Mondes, 15 février 1885, p. 878 (p. 844-881).

44. Jacques de Baudoncourt, Histoire populaire du Canada, d'après les documents français et américains, Paris, Bloud \& Barral, 1888, p. 459 (510 p.). Notons en passant que de Baudoncourt plagie presque l'article (1885) de Du Bled, suivant exactement le même plan, et reproduisant les idées maîtresses au moyen des mêmes expressions et tournures.

45. Charles Gailly de Taurines, la Nation canadienne, Ẽtude historique sur les populations du nord de l'Amérique, Paris, Plon et Nourrit, 1894, 338 p. Voir en particulier les chapitres XIl ( «La langue française au Canada», p. 236-254), XIII ( "La littérature canadienne, les historiens», p. 255-266), XIV (“Romanciers et poètes», p. 267-280). 
46. Eug[ène] Réveillaud, Histoire du Canada et des Canadiens français, de la découverte jusqu'à nos jours, Paris, Grassart, [1884], p. 540 (551 p.).

47. Comte de Toulouse-Lautrec, «A propos d'un livre canadien» [Un pélerinage au pays d'Évangéline], Le Correspondant, CLI, avril-juin 1888, p. I124 (p. 1123-1145). Sera repris en tiré à part, Paris, de Soye et fils, 1888, 27 p.

48. Revue littẻraire (Supplément mensuel du journal L'Union), juillet 1883, p. 111. Le signataire, selon Jean Ménard, pourrait être Nemours-Godré.

49. L[éon] de la Brière, "Causerie littéraire", Samedi-Revue, 1, no 2, 11 février 1888, p. 23 (p. 21-23). Paru, en partie, dans l'Autre France, Voyage au Canada, Paris, E. Dentu, 1886, 151 p. De cet ouvrage, Lanctot dit qu'il ne contient «rien de substantiel ou de particulier" (L'Oeuvre de la France..., p. 158).

50. Rémy de Gourmont, les Canadiens de France, Paris, Firmin-Didot, [1893], p. 210 (256 p.).

51. Sur cette question, on pourrait également consulter Charles Savary, Feuilles volantes. Recueil d'études et d'articles de journaux, Ottawa, 1890, p. 23-24 (517 p.), de même que Frédéric Gerbié, France et Canada, Paris, Charles Schlaeber, 1896, p. 156 (196 p.).

52. R. Martin, Revue du monde catholique, 15 avril 1882 , p. 120 (p. 120-124). Signalons aussi, du même auteur et dans la même revue, des articles parus le 15 juin (sur l'abbé Desmazures) et le 15 août (sur l'abbé Casgrain).

53. Jacques Feyrol, Jes Français en Amérique. Canada-Acadie-Louisiane, Paris, H. Lecène et H. Oudin, [ 1886], p. 105 (240 p.).

54. Napoléon Legendre, «De notre littérature nationale», La Revue des Deux Frances, 1, no 3, 1 er décembre 1897, p. 193 (p. 193-205).

55. Voir la note 4. Comme la présente section ne portera que sur ces deux textes, parus en 1894 et 1895 , nous indiquerons les pages précédées de l'année en question pour signaler l'une ou l'autre étude.

56. Paul Guillaume, le Monde colonial XIXe-XXe siècle, Paris, A. Colin, 1974, p. 34 (296 p.). 